\title{
Seismic wave propagation in anisotropic ice - Part 1: Elasticity tensor and derived quantities from ice-core properties
}

\author{
A. Diez ${ }^{1,2, * * *}$ and O. Eisen $^{1,3}$ \\ ${ }^{1}$ Alfred-Wegener-Institut Helmholtz-Zentrum für Polar- und Meeresforschung, Bremerhaven, Germany \\ ${ }^{2}$ Karlsruher Institut für Technologie, Karlsruhe, Germany \\ ${ }^{3}$ Fachbereich Geowissenschaften, Universität Bremen, Bremen, Germany \\ *now at: Scripps Institution of Oceanography, University of California, San Diego, USA \\ ** Invited contribution by A. Diez, recipient of the EGU Outstanding Student Poster Award 2014.
}

Correspondence to: A. Diez (adiez@ucsd.edu)

Received: 26 May 2014 - Published in The Cryosphere Discuss.: 4 August 2014

Revised: 13 December 2014 - Accepted: 24 December 2014 - Published: 20 February 2015

\begin{abstract}
A preferred orientation of the anisotropic ice crystals influences the viscosity of the ice bulk and the dynamic behaviour of glaciers and ice sheets. Knowledge about the distribution of crystal anisotropy is mainly provided by crystal orientation fabric (COF) data from ice cores. However, the developed anisotropic fabric influences not only the flow behaviour of ice but also the propagation of seismic waves. Two effects are important: (i) sudden changes in COF lead to englacial reflections, and (ii) the anisotropic fabric induces an angle dependency on the seismic velocities and, thus, recorded travel times. A framework is presented here to connect COF data from ice cores with the elasticity tensor to determine seismic velocities and reflection coefficients for cone and girdle fabrics. We connect the microscopic anisotropy of the crystals with the macroscopic anisotropy of the ice mass, observable with seismic methods. Elasticity tensors for different fabrics are calculated and used to investigate the influence of the anisotropic ice fabric on seismic velocities and reflection coefficients, englacially as well as for the ice-bed contact. Hence, it is possible to remotely determine the bulk ice anisotropy.
\end{abstract}

\section{Introduction}

Understanding the dynamic properties of glaciers and ice sheets is one important step to determine past and future behaviour of ice masses. One essential part is to increase our knowledge of the flow of the ice itself. When the ice mass is frozen to the base, its flow is primarily determined by internal deformation. The degree thereof is governed by the viscosity (or the inverse of softness) of ice. The viscosity depends on different factors, such as temperature, impurity content and the orientation of the anisotropic ice crystals (Cuffey and $\mathrm{Pa}$ terson, 2010).

Ice is a hexagonal crystal (ice Ih) under natural conditions on earth. These ice crystals can align in specific directions in response to the stresses within an ice mass. A preferred orientation of the ice crystals causes the complete fabric to be anisotropic, in contrast to a random distribution of the ice crystals where the ice is isotropic on the macroscopic scale. This fabric anisotropy influences the viscosity of the ice. The shear strength is several orders of magnitude smaller perpendicular to the ice crystal's $c$ axis than parallel to it, as shown in laboratory studies (Ashby and Duval, 1985; Cuffey and Paterson, 2010).

The influence of anisotropic ice fabric on the flow behaviour of ice can directly be observed in radar profiles from ice domes. At ice domes and divides a prominent feature of flow conditions is a Raymond bump (Raymond, 1983; Martín et al., 2009b). As ice is a non-Newtonian fluid, it is softer and deforms more easily on the flanks of the ice dome or divide due to the higher deviatoric stress there compared to the centre of the dome. Thus, the vertical flow is slower at the dome itself than on the flanks. This leads to an apparent upwarping of the isochronous layers. The development and influence of anisotropic fabric on the flow of ice at divides and the effects on the development of Raymond bumps were investigated 
by, for instance, Pettit et al. (2007) and Martín et al. (2009a). At ice divides features like double bumps and synclines are observed (Drews et al., 2013), next to single bumps. Martín et al. (2009a) were able to reproduce these double bumps and synclines by including anisotropic rheology in a full-Stokes model. Hence, they are presently considered to be direct evidence of the existence of developed anisotropic fabric.

A second prominent feature in radar data is the basal layer. Before the advent of multi-static, phase-sensitive radar systems, the basal layer had usually been observed only as an echo-free zone (EFZ). The onset of it was connected to the appearance of folds in ice cores on a centimetre scale (Drews et al., 2009). Considerable progress in radar imaging over the last decade make it now possible to image the very bottom layer of ice sheets (Bell et al., 2011; NEEM community members, 2013). The radar data show an often fuzzy basal layer, with a rough upper surface and considerably disturbed coherency of radar return power. The presence of the basal layer turns out to be widespread, especially in Antarctica (CReSIS, P. Gogineni, personal communication, 2014). As the basal ice near the bed is subject to higher stresses and elevated temperatures than the ice above, it is the region where ice physical properties on the microscale change most rapidly (Faria et al., 2014b). These include changes in crystal orientation fabric (COF) properties and distribution.

With increasing computational power the incorporation of anisotropy into ice flow models becomes feasible in three dimensions as well as on regional scales. However, to include anisotropy in ice-flow modelling, we need to understand the development and the distribution of the anisotropic fabric; i.e. we have to observe the variation in the COF distribution over depth, as well as the lateral extent. To extend our ability to determine the influence of these properties on ice flow and map them laterally beyond the $10 \mathrm{~cm}$ scale of ice cores, we have to advance our knowledge of the connection between microscale properties and macroscale features on the scale of tenths of a metre to hundreds of metres observed with geophysical methods like radar and seismics.

The standard method of measuring the COF distribution is to analyse thin sections from ice cores under polarised light. The anisotropy is then normally given in the form of the sample-averaging eigenvalues of the orientation tensor (Woodcock, 1977) in discrete depth intervals. From this we gain information about the local anisotropic conditions at the ice-core location. Radar data have also been used to analyse the changing COF over depth (Matsuoka et al., 2003; Fujita et al., 2006; Eisen et al., 2007; Matsuoka et al., 2009). The challenge in analysing radar data is to distinguish the COFinduced reflections from the numerous conductivity-induced reflections. This distinction is important as conductivityinduced layers are isochrones; by following conductivityinduced reflections in radar data, layers of equal age can be followed over large distances. Currently, identifying and tracing undisturbed layering is one of the main methods be- ing used to identify the location of a site for a potentially 1.5 My old ice core in East Antarctica (Fischer et al., 2013).

Further, the anisotropic fabric has an influence on the wave propagation of seismic waves. Hence, by analysing COFinduced reflections and travel times the anisotropic fabric on the macroscale can be determined. Not only the longitudinal $(\mathrm{P})$ pressure waves but also the transverse waves, i.e. the horizontal (SH) and vertical (SV) shear waves, can be analysed here for the anisotropic fabric. One of the first studies of seismic anisotropy in the context of ice crystal anisotropy was the $\mathrm{PhD}$ thesis of Bennett (1968), who derived equations for the calculation of seismic velocities for solid cone and surface cone fabrics. He fitted curves to the slowness surface (inverse of the phase velocity) calculated from an elasticity tensor measured by means of ultrasonic sounding. This was applied to data from Dome C, Antarctica, by Blankenship and Bentley (1987). Bentley (1972) investigated the anisotropic ice fabric at Byrd Station, Antarctica, for which he used ultrasonic logging. To determine the anisotropic seismic velocities for different cone fabrics, he calculated an average from the single crystal velocity for the encountered directions. This approach was used later by Gusmeroli et al. (2012) for analysing the crystal anisotropy from borehole sonic logging at Dome C, Antarctica.

These methods have one shortcoming. They limit the analysis of anisotropy of seismic waves to the analysis of the travel times, i.e. seismic velocities. The influence of anisotropy has not only been observed in seismic velocities. Englacial reflections were also observed in seismic data from Antarctica (Horgan et al., 2011; Hofstede et al., 2013) and Greenland (Horgan et al., 2008). These reflections were interpreted as arising from an abrupt change in fabric orientation. However, to analyse the reflection signature and determine the actual change in COF, we first need an understanding of the reflection coefficient for changing incoming angles for the transition between different anisotropic fabrics.

One way to improve the analysis of seismic data is to apply full waveform inversion algorithms, i.e. the analysis of the complete observed wave field and not only quantifiable characteristics such as reflection strength or travel times, which is gaining more and more importance in applied geophysics in general. If we want to be able to investigate and understand the influence of the anisotropic ice fabric on the seismic wave field and develop ways to derive information from travel times and reflection signatures about different anisotropic ice fabrics from seismic data, we need to be able to derive the elasticity tensor for different COF distributions.

In this paper we extend the analysis of seismic velocities beyond cone fabrics and derive the elasticity tensor, which is necessary to describe the seismic wave field in anisotropic media. The description of seismic wave propagation in anisotropic materials is based on the elasticity tensor, a 4th-order tensor with 21 unknowns in the general case of anisotropy. If the elasticity tensor is known, seismic velocities, reflection coefficients or reflection angles can be cal- 
culated. From ice core analysis one normally gains the $\mathrm{COF}$ eigenvalues describing the distribution of the crystal orientations. Hence, we first need a connection between the $\mathrm{COF}$ eigenvalues and the elasticity tensor.

We present a framework here to derive the elasticity tensor from the COF eigenvalues for cone as well as different girdle fabrics. We derive opening angles for the enveloping of the $c$ axis distribution from the COF eigenvalues. We then integrate using a monocrystal elasticity tensor for these derived distributions to obtain the elasticity tensor for the different anisotropic fabrics (Sect. 3). Based on these derived elasticity tensors, we calculate seismic velocities and reflection coefficients for different $c$ axis distributions. As examples, we investigate the compressional wave velocity variations with increasing angle for different fabrics and the reflection coefficients for a change from isotropic to girdle fabric for compressional and shear waves. Further, we analyse the influence of anisotropy on the reflection signature of the ice-bed interface and discuss these results in Sect. 4. This is the first part of two companion papers. The calculations introduced here will be applied to ice-core and seismic data from Kohnen Station, Antarctica, in Part II, Diez et al. (2015).

\section{Ice crystal anisotropy}

The ice crystal is an anisotropic, hexagonal crystal with the basal plane perpendicular to the ice crystal's $c$ axis. Due to the existing stresses within glaciers and ice sheets, these anisotropic ice crystals can be forced to align in one or several specific directions. In such cases the crystal's $c$ axis is oriented perpendicular to the main direction of stress (Cuffey and Paterson, 2010). Depending on the stress regime, different COF distributions develop. Common stress regimes in glaciers are simple shear and uniaxial stress (Table 1). At ice domes simple shear can be observed, such that the ice crystals orient towards the vertical; i.e cone distributions can be found, also called cluster distributions in mineralogy. At ice divides, with a main direction of extension and compression perpendicular to that, ice crystals tend to orient in one plane, i.e. in girdle distributions.

Different fabric distributions were discussed by Wallbrecher (1986), who classifies eight different fabric groups. Of these we will use the three most common fabrics observed in glacier ice in the following analysis of the influence of ice crystal anisotropy on seismic wave propagation: (i) the cluster (or cone) distribution, (ii) the thick girdle distribution and (iii) the partial girdle distribution. These distributions are shown in Table 1. The sketches (first row) show the enveloping of the specific $c$ axes' distribution for the different fabrics. We will use the term cone fabric instead of cluster fabric hereinafter, as it is the more commonly used term in glaciology. The most extreme forms of anisotropy we can expect in ice are the isotopic fabric, with a uniform distribution of ice crystals, and the vertical single maximum (VSM) fabric,

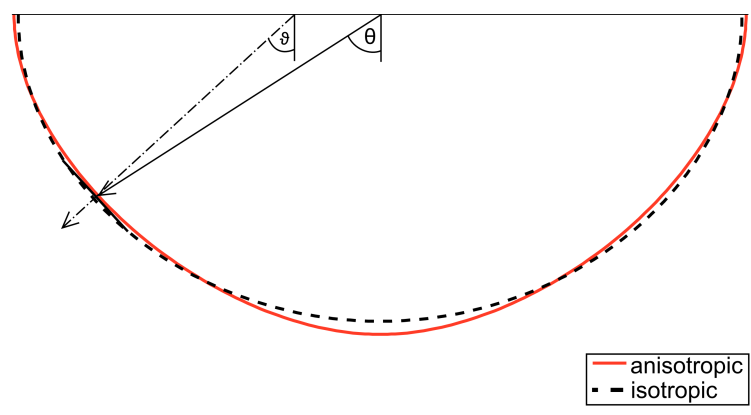

Figure 1. Wavefront of a $\mathrm{P}$ wave travelling in isotropic ice fabric (dashed line) and in a vertical single maximum (VSM) fabric (red line), i.e. a vertical transversely isotropic (VTI) medium. The solid arrow shows the group velocity with group angle $\theta$, the dashed arrow the phase velocity with phase angle $\vartheta$ for the anisotropic case.

where all ice crystals are oriented vertically. Note that the term "lattice-preferred orientation (LPO)" is used as well in the literature to refer to the orientation of the crystals (Faria et al., 2014a), in addition to COF.

\subsection{Crystal orientation fabric measurements}

The standard method of measuring COF distributions is by analysing thin sections from ice cores under polarised light using an automatic fabric analyser (Wilson et al., 2003; Peternell et al., 2010). The $c$ axis orientation of each single crystal is determined and can be given as a unit vector $(\boldsymbol{c})$. These orientations can be presented in Schmidt plots, an equal-area projection of a sphere onto a plane, or as eigenvalues $\lambda_{1}, \lambda_{2}, \lambda_{3}$ of the weighted orientation tensor:

$A_{i j}=W \sum_{l=1}^{n}\left(c_{i} c_{j}\right)_{l}$, with $i, j=1,2,3$.

The number of grains is given by $n$, and $W$ is a weighting function, with weighting e.g. by grain number $(W=1 / n)$ or by area. The three eigenvalues, with $\lambda_{1} \leq \lambda_{2} \leq \lambda_{3}$ and $\sum \lambda_{i}=1$, determine the extension of a rotation ellipsoid. The corresponding eigenvectors cannot be given when the orientation of the ice core within the borehole is not measured in geolocated directions. Hence, the direction to which these eigenvalues apply is often unknown.

Another possibility to describe the anisotropic fabric is to calculate the spherical aperture from the orientation tensor. Hence, the $c$ axis distribution is given in the form of one opening angle for the enveloping cone (Wallbrecher, 1986). However, this limits the analysis of anisotropy to cone fabrics (Table 1).

\subsection{Seismic anisotropy}

The propagation of seismic waves is influenced by the anisotropic material, affecting seismic velocities, reflection coefficients and reflection angles, among other properties. 
Table 1. The different ice crystal distributions as used for the calculation of seismic velocities and reflection coefficients. Given are the sketches for the enveloping of the $c$ axis distribution, the glaciological terms, the common stress regime and the corresponding eigenvalue range. In the second part the seismic term for the anisotropic regime is given together with the opening angles derived from the COF eigenvalues to calculate the elasticity tensor.

\begin{tabular}{|c|c|c|c|c|c|}
\hline \multirow{2}{*}{$\begin{array}{l}\text { Fabrics } \\
\text { Envelope }\end{array}$} & \multicolumn{3}{|c|}{ Glaciological context } & \multicolumn{2}{|c|}{ Seismic context } \\
\hline & Term & Stress regime & Eigenvalues & Term & Opening angle \\
\hline $\mathrm{X}_{2}^{\prime}$ & Isotropic & Uniform & $\lambda_{1}=\lambda_{2}=\lambda_{3}=1 / 3$ & Isotropic & $\varphi=\chi=90^{\circ}$ \\
\hline $\mathrm{X}_{2}^{r^{\prime}}$ & $\begin{array}{l}\text { Cone (cluster in } \\
\text { mineralogy) }\end{array}$ & Simple shear & $\begin{array}{l}\lambda_{1}=\lambda_{2} \\
\lambda_{3} \geq \lambda_{1}, \lambda_{2}\end{array}$ & $\begin{array}{l}\text { Vertical trans- } \\
\text { versely isotropic } \\
\text { (VTI) }\end{array}$ & $\begin{array}{l}\varphi=\chi \\
0^{\circ} \leq \varphi \leq 90^{\circ}\end{array}$ \\
\hline $\mathrm{X}_{2}^{\prime^{\prime}}$ & $\begin{array}{l}\text { Vertical single } \\
\text { maximum (VSM) }\end{array}$ & Simple shear & $\begin{array}{l}\lambda_{1}=\lambda_{2}=0 \\
\lambda_{3}=1\end{array}$ & $\begin{array}{l}\text { Vertical trans- } \\
\text { versely isotropic } \\
\text { (VTI) }\end{array}$ & $\varphi=\chi=0^{\circ}$ \\
\hline
\end{tabular}

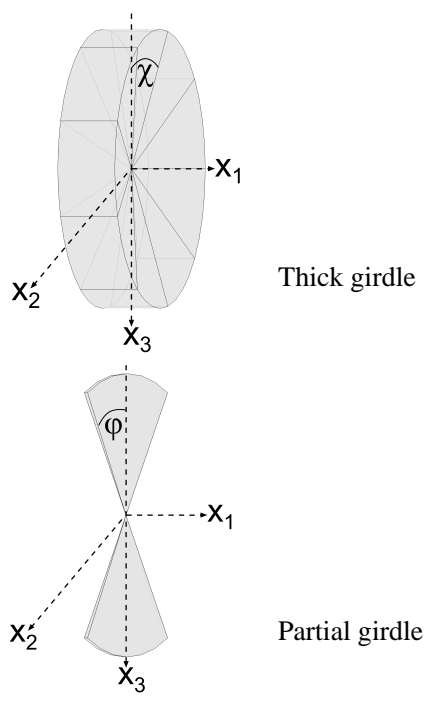

$$
\begin{array}{lll}
\text { Uniaxial compression, }, & \lambda_{2}=\lambda_{3} \\
\text { extension } & \lambda_{1}=1-2 \lambda_{2}
\end{array}
$$

$$
\begin{array}{ll}
\text { Axial compression, } & \lambda_{1}=0 \\
\text { extension } & 0 \leq \lambda_{2} \leq 0.5 \\
& \lambda_{3}=1-\lambda_{2}
\end{array}
$$

Horizontal transversely isotropic (HTI)

$\varphi=90^{\circ}$

$0^{\circ} \leq \chi \leq 90^{\circ}$ 
The propagation of wavefronts in the anisotropic case is no longer spherical. Figure 1 shows the anisotropic wavefront for a $\mathrm{P}$ wave travelling in a VSM fabric (red line) and the spherical wavefront for a $\mathrm{P}$ wave in isotropic ice fabric (dashed black line). For the anisotropic case, group and phase velocity, as well as group angle $\theta$ and phase angle $\vartheta$, are no longer the same. The group velocity determines the travel time. The phase velocity vector is normal to the wavefront. Thus, the phase velocity and phase angle $\vartheta$ are needed for the calculation of reflection and transmission angles as well as reflection coefficients in anisotropic media.

For an anisotropic medium the linear relationship between tensors of stress $\sigma_{m n}$ and strain $\tau_{o p}$ is described by Hooke's law:

$\sigma_{m n}=c_{m n o p} \tau_{o p}$

with the elasticity tensor $c_{m n o p}$ and $m, n, o, p=1,2,3$. In the isotropic case these 81 components of the elasticity tensor can be reduced to the two well-known Lamé parameters. In the general anisotropic case, symmetry considerations of the strain and stress tensors apply, as do thermodynamic considerations (Aki and Richards, 2002). Hence, the general anisotropic elasticity tensor consists of 21 independent components and is referred to as triclinic.

To determine seismic velocities in anisotropic media, a solution for the wave equation needs to be found. Given here is the wave equation for homogeneous, linear elastic media, without external forces and with triclinic anisotropy:

$\rho \frac{\partial^{2} u_{m}}{\partial t^{2}}-c_{m n o p} \frac{\partial^{2} u_{o}}{\partial x_{n} \partial x_{p}}=0$,

with $\rho$ the density of the material, $t$ time, the components $u_{m}$ and $u_{o}$ of the displacement vector $\boldsymbol{u}$ and the different spatial directions $x_{n}, x_{p}$. Solving this equation leads to an eigenvalue problem, the Christoffel equation. For a detailed derivation see, e.g. Tsvankin (2001).

Finally, three non-trivial solutions exist for this eigenvalue problem, giving the three phase velocities and vectors for the quasi compressional (qP), the quasi vertical (qSV) and the quasi horizontal shear (qSH) wave. The phase vectors are orthogonal to each other. However, qP and qSV waves are coupled, so the waves are not necessarily purely longitudinal or shear waves outside of the symmetry planes. Therefore, they are additionally denoted as "quasi" waves, i.e. qP, qSV and qSH waves. As the following analyses are mostly within the symmetry planes, the waves will from now on be denoted as $\mathrm{P}, \mathrm{SV}$ and SH waves. Nevertheless, outside of the symmetry planes this term is not strictly correct.

To be able to find analytical solutions to the Christoffel matrix, the anisotropic materials are distinguished by their different symmetries. Additionally, to simplify calculations with the elasticity tensor, we will use the compressed Voigt notation (Voigt, 1910) for the elasticity tensor $c_{m n o p} \rightarrow C_{i j}$. Therefore, the index combinations of $m n$ and $o p$ are replaced by indices between 1 and $6(11 \equiv 1,22 \equiv 2,33 \equiv 3$,
$23 \equiv 4,13 \equiv 5,12 \equiv 6)$. Considering only certain symmetries reduces the unknowns of the elasticity tensor $C_{i j}$ further. For the analysis of anisotropic ice we consider cone, thick gridle and partial girdle fabric. The connection between the different fabric types and symmetry classes, i.e. seismic terminology for this fabric, can be found in Table 1. Partial girdle fabric is the fabric with the lowest symmetry, corresponding to an orthorhombic medium, with nine unknowns:

$$
C_{i j}=\left(\begin{array}{cccccc}
C_{11} & C_{12} & C_{13} & 0 & 0 & 0 \\
C_{12} & C_{22} & C_{23} & 0 & 0 & 0 \\
C_{13} & C_{23} & C_{33} & 0 & 0 & 0 \\
0 & 0 & 0 & C_{44} & 0 & 0 \\
0 & 0 & 0 & 0 & C_{55} & 0 \\
0 & 0 & 0 & 0 & 0 & C_{66}
\end{array}\right) .
$$

In the case of orthorhombic media three symmetry planes, i.e. orthogonal planes of mirror symmetry, exist. The number of unknowns can be reduced further to five unknowns if transversely isotropic media exist, resulting in an anisotropy with a single axis of rotation symmetry. This is normally distinguished in vertical transversely isotropic (VTI) and horizontal transversely isotropic (HTI) media, with a vertical and horizontal axis of rotation symmetry, respectively. A vertical cone fabric, including VSM fabric, would be classified as VTI medium, while a thick girdle fabric as given in Table 1 would be classified as HTI medium. This distinction is important for the calculation of seismic velocities and reflection coefficients as the calculation simplifies for wave propagation within symmetry planes of the anisotropic fabric (Sect. 4)

\section{Calculation of elasticity tensor from COF eigenvalues}

From the analysis of ice cores we determine the COF eigenvalues which describe the crystal anisotropy over depth. The propagation of seismic waves in anisotropic media can be calculated from the elasticity tensor. Hence, a relationship between the COF eigenvalues and the elasticity tensor is needed.

For the following derivation of the elasticity tensor we will use two opening angles for the description of the fabric that envelopes the $c$ axis distribution. Thus, we are able to take into account cone as well as girdle fabric distributions. We distinguish between an opening angle $\chi$ in $x_{1}$ direction and an opening angle $\varphi$ in $x_{2}$ direction in a coordinate system where the $x_{3}$ axis is pointing downwards (Table 1). These opening angles will be calculated from the COF eigenvalues.

The two opening angles determine the kind of fabric (Table 1). If the angles $\varphi$ and $\chi$ are equal, the $c$ axis distribution is a cone distribution with the cone opening angle $\varphi=\chi$; i.e. it is a VTI medium. The two extrema of this distribution are the uniform distributions, i.e. the isotropic case, and the VSM fabric. All $c$ axes are oriented vertically in the case of a VSM fabric. The eigenvalues are $\lambda_{1}=\lambda_{2}=0$ and $\lambda_{3}=1$, 
and the cone opening angle is $0^{\circ}$. The ice crystals are randomly oriented in the case of isotropic fabric. The eigenvalues are then $\lambda_{1}=\lambda_{2}=\lambda_{3}=1 / 3$, and the cone opening angle is $90^{\circ}$. The thick girdle fabric is an HTI media: the $c$ axes are distributed between two planes with a certain distance, so that the opening angle $\varphi$ in $x_{2}$ direction is $90^{\circ}$ and $\chi$ in $x_{1}$ direction gives the thickness of the girdle. The partial girdle fabric, an orthorhombic medium, is a distribution where all ice crystal $c$ axes are in one plane, but only within a slice of this plane, so that the opening angle $\chi$ in $x_{1}$ direction is $0^{\circ}$ and $\varphi$ in $x_{2}$ direction gives the size of the slice within the plane. A girdle fabric with $\chi=0^{\circ}$ and $\varphi=90^{\circ}$ would correspond to the eigenvalues $\lambda_{1}=0$ and $\lambda_{2}=\lambda_{3}=0.5$.

We will use a measured monocrystal elasticity tensor here to calculate the elasticity tensor for the different observed anisotropic fabrics in ice from the COF eigenvalues. For monocrystalline ice the components of the elasticity tensor have been previously measured by a number of authors with different methods. For the following calculations we use the elasticity tensor of Gammon et al. (1983) $\left(C_{11}=13.93 \pm 0.04 \mathrm{GN} \mathrm{m}^{-2} ; C_{33}=\right.$ $15.01 \pm 0.05 \mathrm{GNm}^{-2} ; \quad C_{55}=3.01 \pm 0.01 \mathrm{GNm}^{-2} ; \quad C_{12}=$ $7.08 \pm 0.04 \mathrm{GN} \mathrm{m}^{-2} ; C_{13}=5.77 \pm 0.02 \mathrm{GN} \mathrm{m}^{-2}$ ). The $c$ axis of this ice crystal is oriented vertically here, parallel to the $x_{3}$ direction (Table 1).

\subsection{From COF eigenvalues to opening angles}

When the COF eigenvalues are derived, the information on the fabric distribution is significantly reduced, especially as the corresponding eigenvectors are normally unknown. Hence, it is not possible to determine the elasticity tensor with at least five unknowns directly from the three $\mathrm{COF}$ eigenvalues. Therefore, we first subdivide the observed anisotropies into different fabric groups (cone, thick girdle and partial girdle fabric) by means of the eigenvalues. Afterwards, we determine their opening angles (Sect. 2.1).

To differentiate between cone and girdle fabric Woodcock (1977) suggests a logarithmic representation of the eigenvalues and classification by a slope

$m=\frac{\ln \left(\lambda_{3} / \lambda_{2}\right)}{\ln \left(\lambda_{2} / \lambda_{1}\right)}$.

The fabric is a cone fabric with $m>1$ and a girdle fabric with $m<1$. However, we want to put a stronger tendency towards a classification of the fabric as cone fabric. In the seismic sense a cone fabric is a VTI medium. It is easier to calculate velocities and reflection coefficients for VTI media compared to girdle fabric, i.e. HTI media. Hence, we use a threshold value to distinguish between cone and girdle fabric. If $\lambda_{1} \leq 0.1$ and $\lambda_{2} \geq 0.2$, the fabric is classified as girdle fabric; everything else is classified as cone fabric. Additionally, we set a threshold to distinguish within the girdle fabric between partial and thick girdle fabric. If $\lambda_{1} \leq 0.05$, the fabric is classified as partial girdle, and otherwise as thick girdle

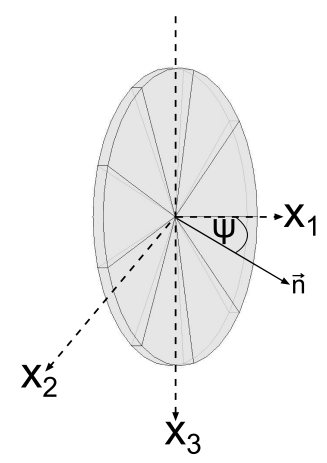

Figure 2. Girdle fabrics classified as HTI media are within the $\left[x_{2}, x_{3}\right]$ plane. When the girdle is rotated around the $x_{3}$ axis, the rotation is given by the azimuth $\psi$.

fabric. By distinguishing between these fabrics we know that $\varphi=\chi$ for the cone fabric, $\varphi=90^{\circ}$ for the thick girdle fabric and $\chi=0^{\circ}$ for the partial girdle fabric (Table 1).

In the next step the remaining, unknown opening angle for the different fabrics needs to be calculated from the eigenvalues, i.e. $\varphi$ for the cone fabric, $\chi$ for the thick girdle fabric and $\varphi$ for the partial girdle fabric. Wallbrecher (1986) for instance connects the opening angle $\varphi$ of a cone fabric with the eigenvalue $\lambda_{3}$ by $\lambda_{3}=1-2 / 3 \sin ^{2} \varphi$. To verify this calculation we determined the eigenvalues for cone angles between 0 and $90^{\circ}$. In total 10000 randomly distributed vectors were created, giving a random distribution of $c$ axes. For each cone angle the vectors within this cone angle were selected. The eigenvalues for this cone angle were then calculated from these vectors. The process was repeated 100 times for each cone angle $\varphi$. The calculated $\lambda_{3}(\varphi)$ values from the equation given by Wallbrecher (1986) differ by up to $15^{\circ}$ for $\varphi$. For a more precise connection of $\lambda_{3}$ and $\varphi$ than available from the literature, a 4th-order polynomial was fitted to the $\lambda_{3}-\varphi$ values (Appendix A1). The same was done for the calculation of $\chi$ from $\lambda_{1}$ for thick girdle fabrics, as well as for the calculation of $\varphi$ from $\lambda_{3}$ for partial girdle fabrics (Appendix A1). The orientation of the girdle is normally not known. Thus, the azimuth $\psi$ (Fig. 2) of the girdle fabric cannot be determined from the eigenvalues. This is only possible if the eigenvector belonging to the eigenvalue $\lambda_{1}$, the normal to the plane of the girdle, is known in geolocated directions. Hence, in the following we normally assume girdle fabrics to be orientated as HTI medium with the azimuth $\psi=0^{\circ}$ for the calculation of the elasticity tensor.

\subsection{From opening angles to the elasticity tensor}

The elasticity tensor of the polycrystal can now be derived using the measured elasticity tensor for a single ice crystal and the derived angles $\chi$ and $\varphi$. For the calculation of the polycrystal elasticity tensor $C_{i j}$ we follow the idea of Nanthikesan and Sunder (1994). They use the concept of the Voigt (1910) and Reuss (1929) bounds. This concept was de- 
veloped to calculate the elasticity tensor of isotropic polycrystals, containing different crystals. This concept is generalised by Nanthikesan and Sunder (1994) to calculate the elasticity tensor for anisotropic fabrics.

Voigt (1910) assumed that the strain on the polycrystal introduces the same uniform strain in all monocrystals. However, Reuss (1929) assumed that the stress on the polycrystal introduces the same uniform stress in all monocrystals. To calculate the elasticity tensor of the polycrystal with the Voigt (1910) assumption, one has to average over the elasticity tensor $C_{i j}^{\mathrm{m}}$ of the monocrystal (superscript m). In the case of the Reuss (1929) assumption, the compliance tensor of the polycrystal is calculated by averaging over the compliance tensor $S_{i j}^{\mathrm{m}}$ of the single crystals. The compliance tensor of a crystal is the inverse of the elasticity tensor, here given in terms of Hooke's law (Eq. 2):

$\tau_{m n}=s_{m n o p} \sigma_{o p}$

For the inversion of elasticity to compliance tensor and vice versa see e.g. Bower (2010). The method of Voigt (1910) and Reuss (1929) is an approximation of the elasticity tensor due to violation of local equilibrium and compatibility conditions across grain boundaries, respectively. Hill (1952) showed that the concepts of Voigt (1910) and of Reuss (1929) give the upper and lower limit for the elastic moduli of the polycrystal $C_{i j}$, referred to as Voigt-Reuss bounds,

$C_{i j}^{\mathrm{R}} \leq C_{i j} \leq C_{i j}^{\mathrm{V}}$

where the superscripts R and V denote the Reuss (1929) and Voigt (1910) calculation, respectively.

To obtain the elasticity tensor of the anisotropic polycrystal $C_{i j}$ from the elasticity tensor of the monocrystal $C_{i j}^{\mathrm{m}}$ with different orientations, one has to integrate the elasticity tensor $\widetilde{C}_{i j}^{\mathrm{m}}(\phi)$ with a probability density function $F(\phi)$ for the different $c$ axes' orientations, where $\phi$ gives the minimum $\left(\phi_{1}\right)$ and maximum $\left(\phi_{2}\right)$ extent of the $c$ axes in the plane. This plane is perpendicular to the corresponding rotation axis, so that the elasticity tensor $\widetilde{C}_{i j}^{\mathrm{m}}(\phi)$ is determined from the monocrystal elasticity tensor $C_{i j}^{\mathrm{m}}$ using the rotation matrix $R_{i j}^{\mathrm{C}}$ :

$\widetilde{C}_{i j}^{\mathrm{m}}(\phi)=\left(R_{i j}^{\mathrm{C}}\right)^{\mathrm{T}} C_{i j}^{\mathrm{m}} R_{i j}^{\mathrm{C}}$.

The rotation matrices $R_{i j}^{\mathrm{C}}$ for the different directions in space are given in Appendix A2; $\left(R_{i j}^{\mathrm{C}}\right)^{\mathrm{T}}$ is the transpose of $R_{i j}^{\mathrm{C}}$. The same applies for the calculation of the monocrystal compliance tensor depending on $\phi$ :

$\widetilde{S}_{i j}^{\mathrm{m}}(\phi)=\left(R_{i j}^{\mathrm{S}}\right)^{\mathrm{T}} S_{i j}^{\mathrm{m}} R^{\mathrm{S}} R_{i j}$,

with the rotation matrix $R_{i j}^{\mathrm{S}}$ for the compliance tensor (Appendix A2) and its transpose $\left(R_{i j}^{\mathrm{S}}\right)^{\mathrm{T}}$. For a uniform distribution of the $c$ axis orientations the probability density function
Table 2. Steps for calculation of elasticity tensor (Eq. 13) or compliance tensor (Eq. 13) for different fabrics (Table 1).

\begin{tabular}{lrrr}
\hline & Step & Rotation axis & Angle \\
\hline \multirow{2}{*}{ Cone } & 1 & $x_{1}$ & $\varphi=\chi$ \\
& 2 & $x_{3}$ & $90^{\circ}$ \\
\hline Partial girdle & 1 & $x_{1}$ & $\varphi$ \\
\hline \multirow{2}{*}{ Thick girdle } & 1 & $x_{1}$ & $90^{\circ}$ \\
& 2 & $x_{2}$ & $\chi$ \\
\hline
\end{tabular}

can be given by

$$
\begin{aligned}
& F(\phi)=\frac{1}{\phi_{2}-\phi_{1}} \quad \text { for } \quad \phi_{1} \leq \phi \leq \phi_{2} \text {, } \\
& =0 \quad \text { for } \quad \phi_{2} \leq \phi \leq \pi ;-\pi \leq \phi \leq \phi_{1} \text {, }
\end{aligned}
$$

which is symmetric around the main orientation, so that $\phi_{1}=$ $-\phi_{0}$ and $\phi_{2}=+\phi_{0}$. The elasticity tensor of the anisotropic polycrystal is then calculated by

$C_{i j}=\frac{1}{2 \phi_{0}} \int_{-\phi_{0}}^{+\phi_{0}} \widetilde{C}_{i j}^{\mathrm{m}}(\phi) \mathrm{d} \phi$,

and the compliance tensor is calculated by

$S_{i j}=\frac{1}{2 \phi_{0}} \int_{-\phi_{0}}^{+\phi_{0}} \widetilde{S}_{i j}^{\mathrm{m}}(\phi) \mathrm{d} \phi$.

After considering the orthorhombic symmetry and some rearranging of the results of Eqs. (12) and (13), the components of the elasticity tensor and compliance tensor of a polycrystal can be expressed in compact form. The results are different for $c$ axes' distributions in the different spatial directions $x_{1}$, $x_{2}$ and $x_{3}$. As an example, the equations for the elasticity and compliance tensor for a rotation around the $x_{1}$ direction are given in Appendix A3. This would correspond to a $c$ axis distribution in the $\left[x_{2}, x_{3}\right]$ plane. The equations for rotation around the $x_{2}$ axis and the $x_{3}$ axis can equally be derived from Eqs. (12) and (13).

The different rotation directions to calculate the polycrystal elasticity tensor $C_{i j}$ from a vertically oriented monocrystal elasticity tensor $C_{i j}^{\mathrm{m}}$ for cone, thick girdle and partial girdle fabric are listed in Table 2. They are also valid for the compliance tensor. For the calculation of the elasticity tensor of a partial girdle (Table 1) the elasticity tensor of the monocrystal $C_{i j}^{\mathrm{m}}$ is rotated around the $x_{1}$ axis with the opening angle of the partial girdle in $x_{2}$ direction $(\varphi)$. The elasticity tensor is then calculated using Eq. (A12) with $\phi_{0}=\varphi$. For a thick girdle, $\varphi=90^{\circ}$ in order to gain a full girdle in the $\left[x_{2}, x_{3}\right]$ plane in the first step. In a second step this elasticity tensor obtained for a full girdle is then rotated around the 
$x_{2}$ axis with $\phi_{0}=\chi$. For cone fabrics with different opening angles the elasticity tensor of a monocrystal is rotated around the $x_{1}$ axis (Eq. A12) in a first step using the cone opening angle $\left(\phi_{0}=\varphi=\chi\right)$; afterwards, the obtained elasticity tensor is rotated around the $x_{3}$ axis with $\phi_{0}=90^{\circ}$.

\subsection{Limitations of the method}

Nanthikesan and Sunder (1994) developed the approach to calculate the polycrystal elasticity tensor from the monocrystal elasticity tensors for what they call S1 (vertical single maximum), S2 (horizontal girdle) and S3 (horizontal partial girdle) ice for given opening angles. They found that the Voigt-Reuss bounds for these fabrics are within $4.2 \%$ of each other, and concluded from this that either calculation, by means of the elasticity tensor (Eq. 12), or compliance tensor (Eq. 13) can be used to calculate the elasticity tensor of the polycrystal. We use the approach of Nanthikesan and Sunder (1994) not only for the calculation of partial girdle fabrics but also for the calculation of the polycrystal elasticity tensor of thick girdle and cone fabrics.

By comparing the individual components of the elasticity tensor derived following Voigt (1910) (Eq. 12) with those of the elasticity tensor derived following Reuss (1929) (Eq. 13 and taking the inverse of the compliance tensor), the largest difference of $4.2 \%$ for all the investigated fabrics can be found for the components $C_{44}\left(S_{44}\right)$ of a partial girdle with an opening angle of 50 and $90^{\circ}$. Thus, for all fabrics in this study, the Voigt-Reuss bounds are within $4.2 \%$ of each other and we follow Nanthikesan and Sunder (1994) in their argumentation that either calculation can be used. However, when using the Voigt (1910) calculation, no extra step in the calculation is needed to invert the compliance tensor. Therefore, for all further calculations the approach by Voigt (1910) is used (Eq. 12).

To be able to calculate the opening angels from the COF eigenvalues, the fabrics are classified into the different fabric groups based on their eigenvalues: cone, thick girdle and partial girdle fabric (Table 1). This classification introduces artificial discontinuities in the velocity profile over depth, calculated from an ice core. These discontinuities only reflect the calculation method, not sudden changes in the prevailing fabric (Part II, Diez et al., 2015). This limitation, introduced by the classification of the different fabric groups, could be overcome by calculating the opening angels directly from the derived $c$ axis vectors. Another possibility would be to calculate the elasticity tensor using the orientation distribution function (ODF), e.g. using the open-source software METX (Mainprice et al., 2011). The calculation of the elasticity tensor in this software is likewise based on Voigt-Reuss bounds, as is done in this study. However, in glaciology the fabric distribution is normally presented in the compact form of the $\mathrm{COF}$ eigenvalues. With the here-presented framework the information of the eigenvalues can directly be used for the calculation of the elasticity tensor, without further information.
To enable direct applicability of our method to existing icecore data sets, we except the limitations of our approach for the sake of ease of use.

For the calculation of the anisotropic polycrystal from the monocrystal neither grain size nor grain boundaries are considered. Elvin (1996) modelled the number of grains that are necessary to homogenise the elastic properties of polycrystalline ice and found that at least 230 grains are needed for girdle fabric (S2 ice). This number of ice crystals should be reached with seismic waves in ice of around $300 \mathrm{~Hz}$, i.e. a wavelength of more than $10 \mathrm{~m}$ and ice crystals with $\leq 0.1 \mathrm{~m}$ diameter on average. Additionally, Elvin (1996) computed two cases, with and without grain boundary sliding, and found a difference of up to $25 \%$ in Young's modulus and the Poisson ratio. In the absence of grain-boundary sliding the anisotropy mainly defines the elastic behaviour. Otherwise, grain shape and grain-boundary sliding become important as well. A certain mistake is, thus, made for the calculation of the polycrystal by only considering the influence of the anisotropy of the monocrystal.

The resultant polycrystal elasticity tensors depend of course on the choice of the monocrystal elasticity tensor. Different authors have measured (Jona and Scherrer, 1952; Green and Mackinnen, 1956; Bass et al., 1957; Brockamp and Querfurth, 1964; Bennett, 1968; Dantl, 1968; Gammon et al., 1983) and calculated (Penny, 1948) the monocrystal elasticity tensor. A comparison of the different elasticity tensors used can be found in Part II (Diez et al., 2015). There we investigate results of a vertical seismic profiling survey in comparison to quantities from measured COF eigenvalues. We find the best agreement between measured and calculated velocities using the monocrystal elasticity tensor of Gammon et al. (1983) for the derivation of the polycrystal elasticity tensor.

\section{Seismic velocities and reflection coefficients in anisotropic ice}

From the derived elasticity tensor we can now calculate velocities and reflection coefficients. Many approximations as well as exact solutions exist for the calculation of velocities and reflection coefficients for different anisotropic fabrics. They are mostly limited to certain symmetries.

In the case of velocities, most studies have been performed on VTI media (e.g. Daley and Heron, 1977). These solutions are still valid within the symmetry planes of HTI media. To be able to calculate seismic velocities for the different fabrics in ice, we will use a calculation of velocities for orthorhombic media derived by Daley and Krebes (2004) (Sect. 4.1). We compare our calculated velocities, based on the derived elasticity tensor, with the well-known velocities for a solid cone that were derived by Bennett (1968) (Sect. 4.2).

For the calculation of the reflection coefficient we use exact (Graebner, 1992) as well as approximate (Rüger, 1997; 
Zillmer et al., 1998) calculations (Sect. 4.3). We show the reflection coefficients for an abrupt change from isotropic to partial girdle fabric here as an example (Sect. 4.4). Additionally, we investigate the influence on the reflection signature of an anisotropic ice mass above the base (Sect. 4.5).

\subsection{Velocities in orthorhombic media}

For the special case of wave propagation in ice with a developed cone fabric anisotropy, Bennett (1968) derived equations of the slowness surface for P, SV and SH waves. The phase velocities are given by the inverse of the slowness surface. To calculate the slowness surface over different angles, Bennett (1968) first derived the elasticity tensor from single natural ice crystals by measurements of ultrasonic pulses of $600 \mathrm{kHz}$. With the derived equations, velocities for different incoming angles $\vartheta$ dependent upon the cone opening angle $\varphi$ can be calculated. It is not possible to calculate velocities for girdle fabrics with this approach.

Using the derived elasticity tensor, we are now able to calculate velocities for different COF distributions. We use the equations derived by Daley and Krebes (2004) for the calculation of phase velocities $v_{\mathrm{ph}}\left(v_{\mathrm{p}}, v_{\mathrm{sv}}, v_{\mathrm{sh}}\right)$ as a function of the phase angle $\vartheta$ for orthorhombic media as given in Appendix B1 (Eqs. B1-B3).

From these phase velocities we have to calculate the group velocities for the calculation of travel times. The calculation of the group velocity vector $\boldsymbol{v}_{\mathrm{g}}$ can be found, e.g., in Rommel and Tsvankin (2000) and Tsvankin (2001). If the propagation of the seismic wave is within symmetry planes of the anisotropic fabric, the group velocity and group angle can be given in compact form. The group velocity $v_{\mathrm{g}}$ is then calculated from the phase velocity $v_{\mathrm{ph}}$ by

$\boldsymbol{v}_{\mathrm{g}}=v_{\mathrm{ph}} \sqrt{1+\left(\frac{1}{v_{\mathrm{ph}}} \frac{\partial v_{\mathrm{ph}}}{\partial \vartheta}\right)^{2}}$

with the group angle $\theta$ in the symmetry plane defined by

$\tan \theta=\frac{\tan \vartheta+\frac{1}{v_{\mathrm{ph}}} \frac{\partial v_{\mathrm{ph}}}{\partial \vartheta}}{1-\frac{1}{v_{\mathrm{ph}}} \frac{\partial v_{\mathrm{ph}}}{\partial \vartheta} \tan \vartheta}$.

Outside the symmetry planes of, e.g., HTI media, all components of the group velocity vector $\boldsymbol{v}_{\mathrm{g}}$ have to be considered (Appendix B1).

Figure 3 shows the phase (dashed curves) and group velocities (solid curves) as a function of the corresponding phase $\vartheta$ and group angle $\theta$ of $\mathrm{P}$ (red), SV (light blue) and SH wave (blue) for a VSM fabric. The largest difference between phase and group velocity can be observed for the SV wave (light blue curves), with a triplication in the group velocity for group angles of $43-47^{\circ}$. Here three different velocities are given for each angle. Due to the small spread of these velocities, we do not expect that this triplication is of relevance for applications given the current day accuracy of measurements. The SV-velocity is largest for $45^{\circ}$ incoming angle

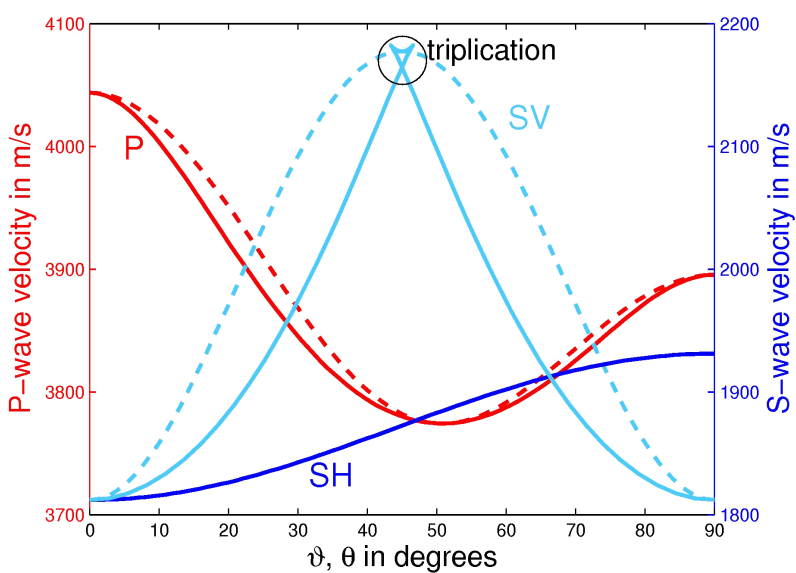

Figure 3. Phase (dashed lines) and group velocities (solid lines) over the corresponding phase $\vartheta$ and group angle $\theta$ for $\mathrm{P}$ (red curves), SH (blue curves) and SV waves (light blue curves) of a VSM fabric. The SV-wave group velocity shows a triplication for group angles $\theta$ between 43 and $47^{\circ}$.

(phase as well as group angle) with $2180 \mathrm{~m} \mathrm{~s}^{-1}$, decreasing for 0 and $90^{\circ}$ to $1810 \mathrm{~m} \mathrm{~s}^{-1}$. Variations for the $\mathrm{SH}$ wave are rather small, with velocities increasing between 0 and $90^{\circ}$ from 1810 to $1930 \mathrm{~m} \mathrm{~s}^{-1}$, i.e. $6 \%$. The $\mathrm{P}$-wave velocity has a minimum at $\sim 51^{\circ}$ incoming angle: $3770 \mathrm{~m} \mathrm{~s}^{-1}$. The highest wave speed is observed for waves parallel to the $c$ axis of an ice crystal $\left(0^{\circ}\right.$ incoming angle) at $4040 \mathrm{~m} \mathrm{~s}^{-1}$, and $150 \mathrm{~m} \mathrm{~s}^{-1}$ $(4 \%)$ slower perpendicular to it.

\subsection{Velocities for anisotropic ice}

By deriving the elasticity tensor for different fabrics, the group and phase velocities of the $\mathrm{P}, \mathrm{SH}$ and $\mathrm{SV}$ wave for these fabrics can now be calculated. Figure 4 show the Pwave phase velocity for different cone and girdle fabrics calculated with the equations given in Daley and Krebes (2004) and the equations derived by Bennett (1968) for a solid cone. The phase velocity for the SH and SV wave as well as the corresponding group velocities can be displayed accordingly (Diez, 2013). Here, we will limit our analysis to $\mathrm{P}$ waves. However, with the derived elasticity tensor, $\mathrm{SH}$ - and $\mathrm{SV}$-wave velocities can just as well be investigated, and the effect of Swave splitting can be analysed.

Figure $4 \mathrm{~d}$ shows the velocities calculated from the equations derived by Bennett (1968) for a solid cone from the elasticity tensor he measured at $-10^{\circ} \mathrm{C}$. These velocities were corrected to $-16^{\circ} \mathrm{C}$ (Kohnen, 1974; Gammon et al., 1983) for better comparison with the other results, where we use the elasticity tensor of Gammon et al. (1983) measured at $-16^{\circ} \mathrm{C}$. The other subfigures are phase velocities calculated with Eq. (12) from an elasticity tensor derived following the steps in Table 2 with the elasticity tensor measured by Gammon et al. (1983). The top row (Fig. 4) shows velocities for cone fabric (subfigure a: VTI) as well as partial girdle fabric 

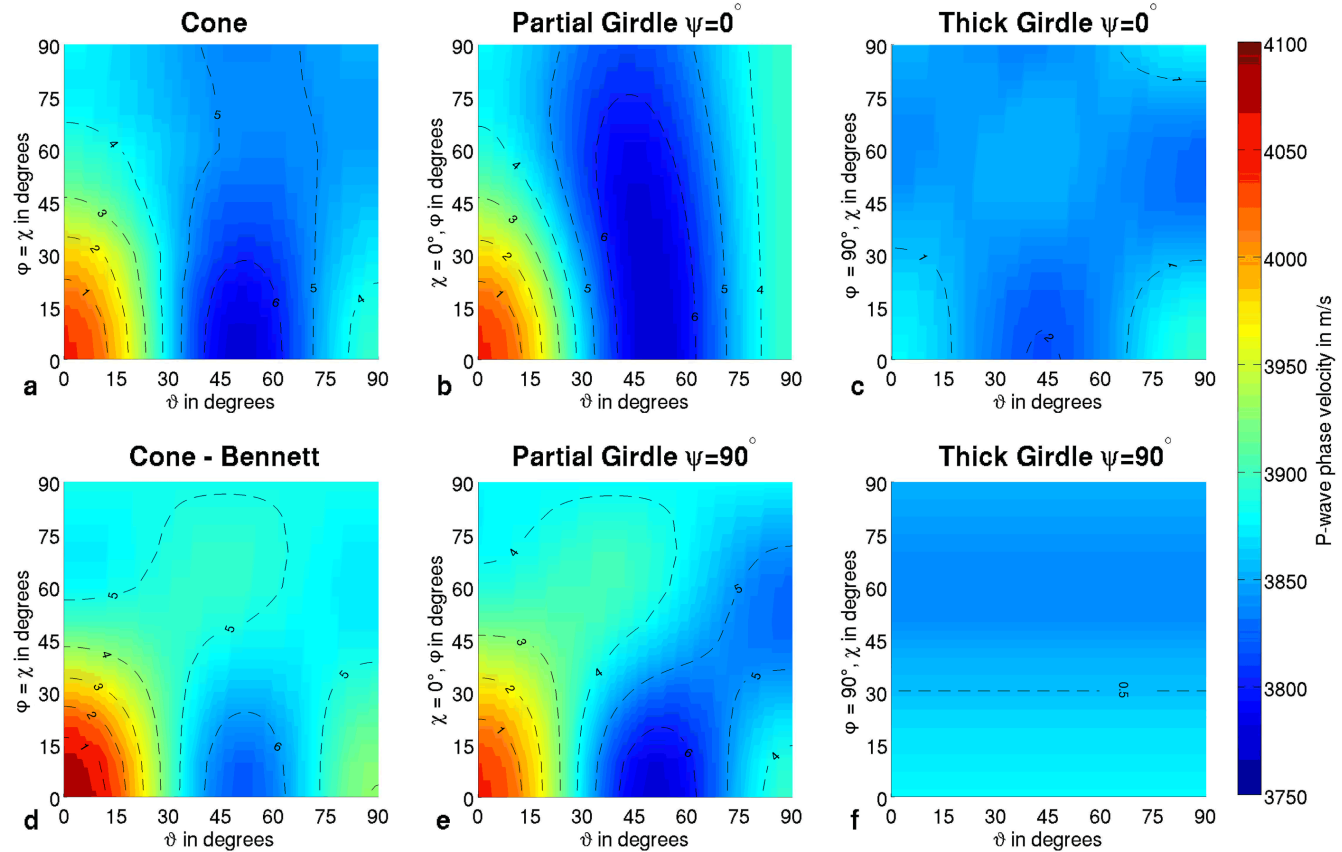

Figure 4. P-wave phase velocities over phase angle $\vartheta$ for different fabrics. P-wave velocity for (a) different cone opening angles $(\varphi=\chi)$, (b) partial girdle fabric $\left(\chi=0^{\circ}\right)$ and (c) thick girdle fabric $\left(\varphi=90^{\circ}\right)$ within the $\left[x_{2}, x_{3}\right]$ plane, (e) partial girdle fabric $\left(\chi=0^{\circ}\right)$ and $(\mathbf{f})$ thick girdle fabric $\left(\varphi=90^{\circ}\right)$ within the $\left[x_{1}, x_{3}\right]$ plane calculated with Eq. (B1) given by Daley and Krebes (2004). (d) shows the P-wave velocity for different cone opening angles $(\varphi=\chi)$ calculated with the equation given by Bennett (1968). The contour lines give the velocity differences in percent, in relation to the maximum velocity of the respective fabric group.

(b: HTI) and thick girdle fabric (c: HTI) in the $\left[x_{2}, x_{3}\right]$ plane, while the bottom row shows velocities for cone fabric calculated following Bennett (1968) (d: VTI) as well as partial girdle fabric (f: $\psi=90^{\circ}$ ) and thick girdle fabric (e: $\psi=90^{\circ}$ ) in the $\left[x_{1}, x_{3}\right]$ plane.

The partial girdle $\left(\chi=0^{\circ}\right.$, Fig. 4 , b, e) with $\varphi=90^{\circ}$ displays the same fabric as the thick girdle $\left(\varphi=90^{\circ}\right.$, Fig. 4 , c, f) with $\chi=0^{\circ}$. The same applies to the cone fabric with an opening angle of $90^{\circ}$ (Fig. 4, a, d) as well as the thick girdle fabric $\left(\varphi=90^{\circ}\right)$ with $\chi=90^{\circ}$ (Fig. $4 \mathrm{c}$, f), both showing isotropic $c$ axes' distribution. Apart from Bennett's velocities, these velocities for the isotropic state (Fig. 4a, c, f) are obviously not isotropic. Slight variations still exist for these velocities with increasing incoming angle. This is due to artefacts that seem to appear from the derivation of the elasticity tensor for the isotropic state using the single crystal elasticity tensor.

It should also be noted that for a thick girdle with $\varphi=\chi=90^{\circ}$ the variations over the incoming angle are just reversed to those of the cone fabric with opening angle $\varphi=\chi=90^{\circ}$. This reflects the difference in the calculation of the elasticity tensor from cone fabric and girdle fabric. While a girdle with $\varphi=90^{\circ}\left(\chi=0^{\circ}\right)$ is calculated in the first step for both fabrics (Table 2) by integration with rotation around the $x_{1}$ axis, the second step is an integration with rotation around the $x_{3}$ axis for the cone fabric and around the $x_{2}$ axis for the thick girdle fabric.
The higher velocities calculated with the equations of Bennett (1968) (Fig. 4, d) are due to the difference in the elasticity tensor, as the elasticity tensor derived by Gammon et al. (1983) was used for the calculation in all of the other subfigures (Fig. 4a-c, e, f). The Bennett (1968) calculation exhibits an isotropic state for $\varphi=\chi=90^{\circ}$. However, this is only possible as Bennett (1968) used fitted curves for the derivation of the slowness surface.

\subsection{Reflection coefficients}

The calculation of reflection coefficients for different incoming angles is already rather complicated for layered isotropic media given by the Zoeppritz equations (e.g. Aki and Richards, 2002). In the case of anisotropic media most of the studies have been done for VTI media (Keith and Crampin, 1977; Daley and Heron, 1977) and in terms of Thomsen parameters (Thomsen, 1993). A comprehensive overview of the different calculations of reflection coefficients for VTI and HTI media is given by Rüger (2002).

In the following, we use equations derived by Zillmer et al. (1997) by means of perturbation theory for the calculation of englacial reflection horizons. These equations for general anisotropy were simplified by Zillmer et al. (1998) for weak contrast interfaces. They are, thus, especially practical for the reflection coefficients in ice. For the isotropic reference values the elasticity tensor for isotropic ice can be used and no 
average needs to be taken over different materials. The reflection coefficients for the anisotropic material are then calculated as perturbations of the isotropic ice fabric. Thus, reflection coefficients for P, SV and SH waves are obtained. The equations for the calculation of reflection coefficients are given in Appendix B2. The $R_{\text {shsh }}$ and $R_{\text {svsv }}$ reflection coefficients are restricted to a symmetry plane of the layered medium. The indices give the polarisation of the incoming and reflected wave; e.g. $R_{\mathrm{pp}}$ is the reflection coefficient for an incoming $\mathrm{P}$ wave, reflected as $\mathrm{P}$ wave, equivalent for $R_{\text {shsh }}$ and $R_{\text {svsv }}$.

To calculate the P-wave reflection coefficient for the bed reflector with an overlaying cone fabric, i.e. VTI media, we use the equations given by Thomsen (1993), which were further developed by Rüger (1997). Exact solutions for VTI media are, for example, given by Keith and Crampin (1977) and Graebner (1992).

\subsection{Reflection coefficients for anisotropic ice}

With the equations given in Appendix B2 (Zillmer et al., 1998) reflection coefficients can be calculated for interfaces between different fabrics. Figure 5 shows as an example the $R_{\mathrm{pp}}, R_{\text {shsh }}$ and $R_{\text {svsv }}$ reflection coefficient for the transition at a layer interface from an isotropic fabric to a partial girdle fabric, both for HTI media $\left(\psi=0^{\circ}\right)$ and with an azimuth of $\psi=90^{\circ}$.

The reflection coefficients are given for angles of incidence between 0 and $60^{\circ}$. This has two reasons. Firstly, most seismic surveys do not exceed an incoming angle of $60^{\circ}$ as this already corresponds to a large offset compared to the probed depth. Secondly and more important, the calculation of the reflection coefficients using Eqs. (B12)-(B13) is not exact. Instead, the error increases with increasing incoming angle.

The largest magnitude of reflection coefficients can be observed for the SVSV reflection (Fig. 5). However, the reflection coefficients are $\leq 0.1$ for all fabric combinations shown here. Most significantly, for the PP reflection the reflection coefficients between different anisotropic fabrics are small. The PP reflection between, for example, isotropic and VSMfabric ice for normal incidence is $<0.02$. For comparison the reflection coefficient between isotropic and lithified sediments (Fig. 6) is $\sim 0.4$. Hence, reflection coefficients at the ice-bed interface are an order of magnitude larger than reflection coefficients for the transition between different anisotropic fabrics. To be able to observe englacial seismic reflections, abrupt changes (i.e. within a wavelength) with significant variations in the orientation of the ice crystals are needed. Such englacial reflections have been observed in data from Greenland (Horgan et al., 2008) and Antarctica (Horgan et al., 2011; Hofstede et al., 2013), and also in the Swiss/Italian Alps (Polom et al., 2014; Diez et al., 2013). These reflections can indicate a change in the fabric. However, the investigation of reflection signatures (amplitude ver-
Table 3. P-wave velocity, S-wave velocity and density for different bed scenarios and isotropic ice as given in Peters et al. (2008). These values are used for the calculation of reflection coefficients given in Fig. 6.

\begin{tabular}{lrrr}
\hline Material & $v_{\mathrm{p}}$ in $\mathrm{m} \mathrm{s}^{-1}$ & $v_{\mathrm{s}}$ in $\mathrm{m} \mathrm{s}^{-1}$ & $\rho$ in $\mathrm{kg} \mathrm{cm}^{-3}$ \\
\hline Ice & 3810 & 1860 & 920 \\
Basement & 5200 & 2800 & 2700 \\
Lithfied sediment & 3750 & 2450 & 2450 \\
Dilatant sediment & 1700 & 200 & 1800 \\
Water & 1498 & 0 & 1000 \\
\hline
\end{tabular}

sus offset, AVO) of englacial reflectors is difficult due to the small reflection coefficients, and the small range they cover with changing incoming angle.

For englacial reflections caused by changing $\mathrm{COF}$, the variations in the reflection coefficient with offset are very small: the PP-reflection coefficient for the transition from isotropic to VSM fabric $\left(\varphi=0^{\circ}\right.$, Fig. 5) from 0 to $60^{\circ}$ is between 0.019 and 0.036 . To put these values in perspective, we consider error bars for reflection coefficients as determined for ice-bed interfaces. It cannot be expected that the error bars for measuring the reflection coefficient of englacial reflections would be smaller than those given for the bed reflection coefficients. Peters et al. (2008) analysed the reflection amplitude for the ice-bed interface from a survey near the South Pole. For the reflection coefficients they derive from the seismic data, they give error bars of \pm 0.04 , with increasing error bars for decreasing incoming angles, limited by \pm 0.2 . The change in the reflection coefficient with offset for englacial reflection that we calculate is smaller than the given error bars. Thus, it is not possible to derive information about the anisotropic fabric from englacial reflections using AVO analysis, at the moment. To be able to derive fabric information from AVO analysis the error in determining the reflection coefficient from seismic data needs to be reduced, e.g. better shooting techniques to reduce the signal-to-noise ratio (SNR) in the data or a better understanding of the source amplitude as well as the damping of seismic waves in ice.

\subsection{Reflection coefficients for ice-bed interface}

Of special interest is the determination of the properties of the ice-bed interface from seismic data. It is possible to determine the bed properties below an ice sheet or glacier by analysing the normal incident reflection coefficient (e.g. Smith, 2007) or by AVO analysis (Anandakrishnan, 2003; Peters et al., 2008). Figure 6 shows reflection coefficients for the transition from isotropic and anisotropic (VSM fabric) ice to different possible bed properties (Table 3). The values for density, P-wave and S-wave velocity, for the different bed scenarios and the isotropic ice, are taken from Peters et al. (2008). For the anisotropic VSM fabric the elasticity tensor of Gammon et al. (1983) is used. 

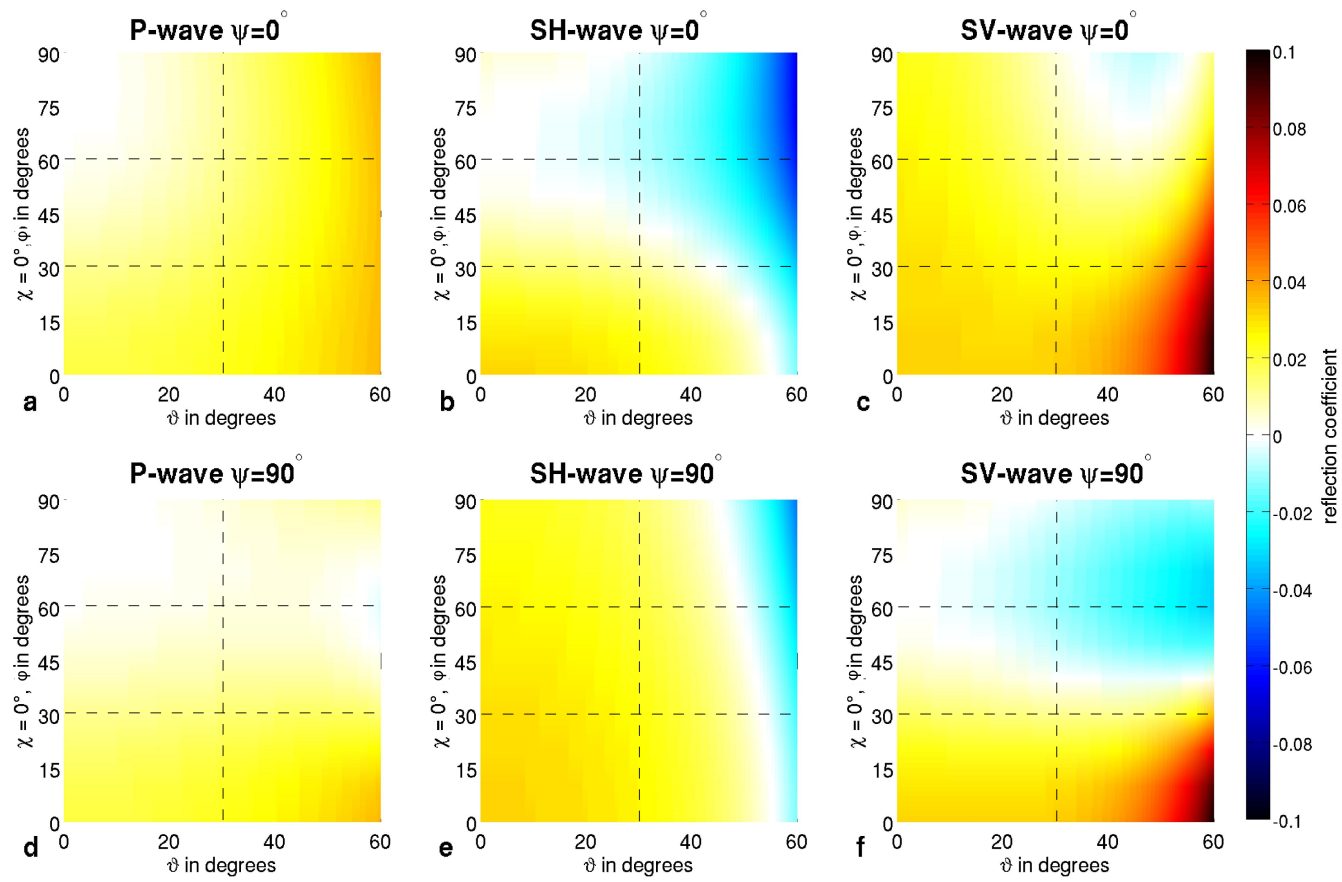

Figure 5. Reflection coefficients for the boundary between an isotropic (upper) layer and a partial girdle fabric (lower) layer with different opening angles $\varphi\left(\chi=0^{\circ}\right)$ of the girdle. The reflection coefficients are calculated with equations given in Sect. 4.3 for different incoming phase angles $\vartheta$. The subfigures (a), (b) and (c) show the reflection coefficients for a girdle orientation (lower layer) perpendicular to the travel path of the wave (HTI media) for PP, SHSH and SVSV reflection, respectively. The subfigures (d), (e) and (f) show the reflection coefficients for a girdle orientation parallel to the travel path of the wave (azimuth $\psi=90^{\circ}$ ) for PP, SHSH and SVSV reflection, respectively.

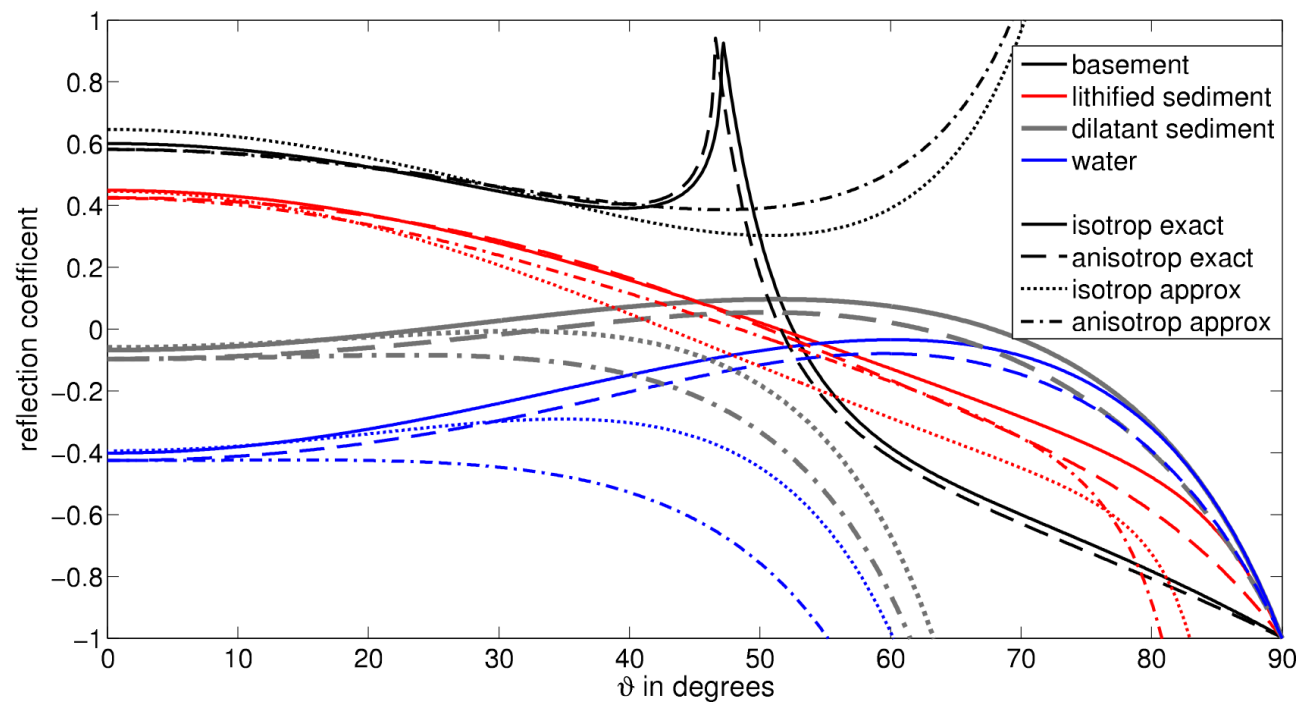

Figure 6. P-wave reflection coefficients for ice-bed interface with different bed properties as a function of phase angle of incidence $\vartheta$ : basement (black), lithified sediments (red), dilatant sediments (gray) and water (blue). The solid and dotted lines are the reflection coefficients for an isotropic ice overburden, the dashed and dashed-dotted lines for the anisotropic (VSM) overburden. The solid and dashed lines are the reflection coefficients calculated with exact equations for VTI media given by Graebner (1992) and Rüger (2002). The dotted and dasheddotted lines are approximate calculations following the approach by Aki and Richards (2002) for the isotropic case and that of Rüger (1997) for the anisotropic case, respectively. Property values for the bed and isotropic ice are taken from Peters et al. (2008). For the anisotropic ice the elasticity tensor given by Gammon et al. (1983) is used. 
Exact solutions are calculated using the equations given by Graebner (1992), with corrections by Rüger (2002). Their equations were used to calculate the exact reflection coefficients for the isotropic ice above the bed (solid lines) and for the anisotropic ice above the bed (dashed lines) shown in Fig. 6. The approximate reflection coefficients for the isotropic ice above the bed (dotted lines) are calculated using equations given in Aki and Richards (2002). The approximate reflection coefficients for the VSM fabric above the bed (dashed-dotted lines) are calculated using equations given in Rüger (1997).

The differences between the isotropic (solid lines) and anisotropic reflection coefficients (dashed lines) are small $(\leq 0.04)$ for the exact solutions. The approximate calculations fit well to the exact solutions up to a group angle of about $30^{\circ}$, with differences of the same order as isotropic to anisotropic variations. However, differences between exact and approximate reflection coefficients become large for increasing phase angle $\left(\geq 30^{\circ}\right)$. Thus, errors introduced by using approximate calculations for the reflection coefficients are larger than the effect of anisotropic ice fabric above the bed.

The observable differences of reflection coefficients for an isotropic and a VSM-fabric overburden are $\leq 0.04$, i.e. smaller then the smallest error bars given by Peters et al. (2008) (Sect. 4.4). The VSM fabric is the strongest anisotropy to be expected in ice. If an anisotropic layer exists above the bed, it yields a different reflection coefficient compared to the case of the isotropic ice overburden. However, the difference between the isotropic overburden reflection coefficient and the anisotropic overburden reflection coefficient is within the range of the error bars given by Peters et al. (2008). Thus, the anisotropic fabric will not have a measurable influence on the analysis of the bed properties by means of the AVO method, given the current degree of data accuracy and SNR.

\section{Conclusions}

We presented an approach to derive the ice elasticity tensor, required for the calculation of seismic wave propagation in anisotropic material, from the $\mathrm{COF}$ eigenvalues derived from ice-core measurements. From the elasticity tensors we derived seismic phase and group velocities of $\mathrm{P}, \mathrm{SH}$ and $\mathrm{SV}$ waves for cone, partial girdle and thick girdle structures, i.e. orthorhombic media. Velocities we derived for different cone fabrics agree well with velocities derived for cone fabric using the already-established method of Bennett (1968). However, with our method it is now also possible to calculate velocities for girdle fabrics. Further, we can use the derived elasticity tensors to investigate the reflections coefficients in anisotropic ice.
We used the elasticity tensor to derive the reflection signature for englacial fabric changes and investigated the influence of anisotropic fabric on the reflection coefficients for basal reflectors. We found that the reflection coefficients and the variations of the reflection coefficients with increasing offset are weak for the transition between different COF distributions: they are at least an order of magnitude smaller than reflections from the ice-bed interface. Thus, either significant changes in the COF distribution or extremely sensitive measurement techniques are needed to observe englacial seismic reflections. The influence of anisotropic ice fabric compared to the isotropic case for the reflection at the icebed interface is so small that it is within the measurement inaccuracy of currently employed seismic AVO analysis. An important result is that the difference between exact and approximate calculations of reflection coefficients for the icebed interface is larger than the influence of an anisotropic ice fabric above the bed. This implies that exact calculations are necessary if the fabric above the bed is in the focus of AVO analysis.

Better results in the calculation of the elasticity tensor could probably be gained by calculation of the opening angles directly from the $c$ axes' vectors. This would avoid our classification into cone, partial girdle and thick girdle fabric. Nevertheless, the approach presented here offers the opportunity to use the readily available COF data from ice cores and go towards an investigation of the seismic wave field in ice without the limitation to velocities only. The inclusion of further properties influencing the propagation of seismic waves in ice, like density and temperature, will offer the opportunity to model the complete wave field. Hence, we are confident that it will become feasible in the future to derive physical properties of the ice from analyses of the complete observed wave field by full waveform inversions. 
Appendix A: From COF eigenvalues to elasticity tensor for seismics

\section{A1 Connection of eigenvalues to opening angles}

The following equations give the connection between the eigenvalues $\lambda_{1}, \lambda_{2}$ and $\lambda_{3}$ and the two opening angles $\varphi$ and $\chi$.

For a cone fabric the angle $\varphi=\chi$ is calculated by

$$
\begin{aligned}
\varphi & =\chi=b_{1} \sin \left(c_{1} \lambda_{3}+d_{1}\right)+b_{2} \sin \left(c_{2} \lambda_{3}+d_{2}\right) \\
& +b_{3} \sin \left(c_{3} \lambda_{3}+d_{3}\right)+b_{4} \sin \left(c_{4} \lambda_{3}+d_{4}\right),
\end{aligned}
$$

with

$b_{1}=141.9, c_{1}=6.251, d_{1}=2.157$,

$b_{2}=139, c_{2}=10.33, d_{2}=-1.809$,

$b_{3}=90.44, c_{3}=14.68, d_{3}=4.685$,

$b_{4}=36.61, c_{4}=16.9, d_{4}=12.63$.

For a thick girdle fabric the angle $\chi$ is calculated by

$$
\begin{aligned}
\chi= & p_{1} \lambda_{1}^{7}+p_{2} \lambda_{1}^{6}+p_{3} \lambda_{1}^{5}+p_{4} \lambda_{1}^{4}+p_{5} \lambda_{1}^{3}+p_{6} \lambda_{1}^{2} \\
& +p_{7} \lambda_{1}+p_{8},
\end{aligned}
$$

$\varphi=90^{\circ}$,

with

$$
\begin{aligned}
& p_{1}=2.957 \times 10^{7}, p_{2}=-3.009 \times 10^{7}, p_{3}=1.233 \times 10^{7}, \\
& p_{4}=-2.599 \times 10^{6}, \\
& p_{5}=3.023 \times 10^{5}, p_{6}=-1.965 \times 10^{4}, p_{7}=877.6, \\
& p_{8}=2.614
\end{aligned}
$$

For a partial girdle fabric the angle $\varphi$ is calculated by

$$
\begin{aligned}
\varphi & =a_{1} \sin \left(b_{1} \lambda_{3}+c_{1}\right)+a_{2} \sin \left(b_{2} \lambda_{3}+c_{2}\right) \\
& +a_{3} \sin \left(b_{3} \lambda_{3}+c_{3}\right)+a_{4} \sin \left(b_{4} \lambda_{3}+c_{4}\right), \\
\chi & =0^{\circ},
\end{aligned}
$$

with

$a_{1}=118.7, b_{1}=7.415, c_{1}=-3.517$,

$a_{2}=97.47, b_{2}=13.68, c_{2}=1.161$,

$a_{3}=46.57, b_{3}=18.58, c_{3}=6.935$,

$a_{4}=7.455, b_{4}=25.18, c_{4}=11.47$.

\section{A2 Rotation matrices for elasticity and compliance tensor}

Here the rotation matrix for the elasticity tensor and compliance tensor following Sunder and Wu (1994) are given. For the calculation of the elasticity tensor for different fabrics the monocrystal elasticity tensor needs to be rotated (Sect. 3.2).
The rotation matrix for the elasticity tensor is

$\mathbf{R}^{\mathrm{C}}=\left(\begin{array}{cccccc}l_{1}^{2} & m_{1}^{2} & n_{1}^{2} & 2 m_{1} n l_{1} & 2 n_{1} l_{1} & 2 l_{1} m_{1} \\ l_{2}^{2} & m_{2}^{2} & n_{2}^{2} & 2 m_{2} n l_{2} & 2 n_{2} l_{2} & 2 l_{2} m_{2} \\ l_{3} 1^{2} & m_{3}^{2} & n_{3}^{2} & 2 m_{3} n l_{3} & 2 n_{3} l_{3} & 2 l_{3} m_{3} \\ l_{2} l_{3} & m_{2} m_{3} & n_{2} n_{3} & m_{2} n_{3}-m_{3} n_{2} & n_{2} l_{3}-n_{3} l_{2} & l_{2} m_{3}-l_{3} m_{2} \\ l_{3} l_{1} & m_{3} m_{1} & n_{3} n_{1} & m_{3} n_{1}-m_{1} n_{3} & n_{3} l_{1}-n_{1} l_{3} & l_{3} m_{1}-l_{1} m_{3} \\ l_{1} l_{2} & m_{1} m_{2} & n_{1} n_{2} & m_{1} n_{2}-m_{2} n_{1} & n_{1} l_{2}-n_{2} l_{1} & l_{1} m_{2}-l_{2} m_{1}\end{array}\right)$,

and the compliance tensor

$\mathbf{R}^{\mathrm{S}}=\left(\begin{array}{cccccc}l_{1}^{2} & m_{1}^{2} & n_{1}^{2} & m_{1} n l_{1} & n_{1} l_{1} & l_{1} m_{1} \\ l_{2}^{2} & m_{2}^{2} & n_{2}^{2} & m_{2} n l_{2} & n_{2} l_{2} & l_{2} m_{2} \\ l_{3} 1^{2} & m_{3}^{2} & n_{3}^{2} & m_{3} n l_{3} & n_{3} l_{3} & l_{3} m_{3} \\ 2 l_{2} l_{3} & 2 m_{2} m_{3} & 2 n_{2} n_{3} & m_{2} n_{3}-m_{3} n_{2} & n_{2} l_{3}-n_{3} l_{2} & l_{2} m_{3}-l_{3} m_{2} \\ 2 l_{3} l_{1} & 2 m_{3} m_{1} & 2 n_{3} n_{1} & m_{3} n_{1}-m_{1} n_{3} & n_{3} l_{1}-n_{1} l_{3} & l_{3} m_{1}-l_{1} m_{3} \\ 2 l_{1} l_{2} & 2 m_{1} m_{2} & 2 n_{1} n_{2} & m_{1} n_{2}-m_{2} n_{1} & n_{1} l_{2}-n_{2} l_{1} & l_{1} m_{2}-l_{2} m_{1}\end{array}\right)$

with the direction cosines

$\left(\begin{array}{ccc}l_{1} & l_{2} & l_{3} \\ m_{1} & m_{2} & m_{3} \\ n_{1} & n_{2} & n_{3}\end{array}\right)$

for rotation around the $x_{1}$ axis,

$\left(\begin{array}{ccc}1 & 0 & 0 \\ \cos \phi & -\sin \phi & 0 \\ \sin \phi & \cos \phi & 0\end{array}\right)$

for rotation around the $x_{2}$ axis,

$\left(\begin{array}{ccc}\cos \phi & 0 & -\sin \phi \\ 0 & 1 & 0 \\ \sin \phi & 0 & \cos \phi\end{array}\right)$

and for rotation around the $x_{3}$ axis,

$\left(\begin{array}{ccc}\cos \phi & -\sin \phi & 0 \\ \sin \phi & \cos \phi & 0 \\ 0 & 0 & 1\end{array}\right)$

\section{A3 Components of elasticity and compliance tensor for polycrystal}

The components of the polycrystal elasticity tensor as derived from Eq. (12) with $c$ axes' distribution around the 
$x_{1}$ axis, i.e. within the $\left[x_{2}, x_{3}\right]$ plane, are calculated by

$C_{11}^{\mathrm{p}}=C_{11}^{\mathrm{m}}$,

$C_{22}^{\mathrm{p}}=\frac{1}{2 \phi_{0}}\left[b_{1} C_{22}^{\mathrm{m}}+b_{2} C_{33}^{\mathrm{m}}+2 b_{3}\left(C_{23}^{\mathrm{m}}+2 C_{44}^{\mathrm{m}}\right)\right]$,

$C_{33}^{\mathrm{p}}=\frac{1}{2 \phi_{0}}\left[b_{1} C_{33}^{\mathrm{m}}+b_{2} C_{22}^{\mathrm{m}}+2 b_{3}\left(C_{23}^{\mathrm{m}}+2 C_{44}^{\mathrm{m}}\right)\right]$,

$C_{44}^{\mathrm{p}}=\frac{1}{2 \phi_{0}}\left[\left(b_{1}+b_{2}\right) C_{44}^{\mathrm{m}}+b_{3}\left(C_{22}^{\mathrm{m}}-2 C_{23}^{\mathrm{m}}+C_{33}^{\mathrm{m}}-2 C_{44}^{\mathrm{m}}\right)\right]$,

$C_{55}^{\mathrm{p}}=\frac{1}{2 \phi_{0}}\left[C_{55}^{\mathrm{m}}\left(\phi_{0}+\alpha\right)+C_{66}^{\mathrm{m}}\left(\phi_{0}-\alpha\right)\right]$,

$C_{66}^{\mathrm{p}}=\frac{1}{2 \phi_{0}}\left[C_{66}^{\mathrm{m}}\left(\phi_{0}+\alpha\right)+C_{55}^{\mathrm{m}}\left(\phi_{0}-\alpha\right)\right]$,

$C_{12}^{\mathrm{p}}=\frac{1}{2 \phi_{0}}\left[C_{12}^{\mathrm{m}}\left(\phi_{0}+\alpha\right)+C_{13}^{\mathrm{m}}\left(\phi_{0}-\alpha\right)\right]$,

$C_{13}^{\mathrm{p}}=\frac{1}{2 \phi_{0}}\left[C_{13}^{\mathrm{m}}\left(\phi_{0}+\alpha\right)+C_{12}^{\mathrm{m}}\left(\phi_{0}-\alpha\right)\right]$,

$C_{23}^{\mathrm{p}}=\frac{1}{2 \phi_{0}}\left[\left(b_{1}+b_{2}\right) C_{23}^{\mathrm{m}}+b_{3}\left(C_{22}^{\mathrm{m}}-4 C_{44}^{\mathrm{m}}+C_{33}^{\mathrm{m}}\right)\right]$.

The components of the polycrystal compliance tensor as derived from Eq. (13) with $c$ axes' distribution around the $x_{1}$ axis, i.e. within the $\left[x_{2}, x_{3}\right]$ plane, are calculated by

$S_{11}^{\mathrm{p}}=S_{11}^{\mathrm{m}}$,

$S_{22}^{\mathrm{p}}=\frac{1}{2 \phi_{0}}\left[b_{1} S_{22}^{\mathrm{m}}+b_{2} S_{33}^{\mathrm{m}}+b_{3}\left(2 S_{23}^{\mathrm{m}}+S_{44}^{\mathrm{m}}\right)\right]$,

$S_{33}^{\mathrm{p}}=\frac{1}{2 \phi_{0}}\left[b_{1} S_{33}^{\mathrm{m}}+b_{2} S_{22}^{\mathrm{m}}+b_{3}\left(2 S_{23}^{\mathrm{m}}+S_{44}^{\mathrm{m}}\right)\right]$,

$S_{44}^{\mathrm{p}}=\frac{1}{2 \phi_{0}}\left[\left(b_{1}+b_{2}\right) S_{44}^{\mathrm{m}}+4 b_{3}\left(S_{22}^{\mathrm{m}}-2 S_{23}^{\mathrm{m}}+S_{33}^{\mathrm{m}}-\frac{1}{2} S_{44}^{\mathrm{m}}\right)\right]$,

$S_{55}^{\mathrm{p}}=\frac{1}{2 \phi_{0}}\left[S_{55}^{\mathrm{m}}\left(\phi_{0}+\alpha\right)+S_{66}^{\mathrm{m}}\left(\phi_{0}-\alpha\right)\right]$,

$S_{66}^{\mathrm{p}}=\frac{1}{2 \phi_{0}}\left[S_{66}^{\mathrm{m}}\left(\phi_{0}+\alpha\right)+S_{55}^{\mathrm{m}}\left(\phi_{0}-\alpha\right)\right]$,

$S_{12}^{\mathrm{p}}=\frac{1}{2 \phi_{0}}\left[S_{12}^{\mathrm{m}}\left(\phi_{0}+\alpha\right)+S_{13}^{\mathrm{m}}\left(\phi_{0}-\alpha\right)\right]$,

$S_{13}^{\mathrm{p}}=\frac{1}{2 \phi_{0}}\left[S_{13}^{\mathrm{m}}\left(\phi_{0}+\alpha\right)+S_{12}^{\mathrm{m}}\left(\phi_{0}-\alpha\right)\right]$,

$S_{23}^{\mathrm{p}}=\frac{1}{2 \phi_{0}}\left[\left(b_{1}+b_{2}\right) S_{23}^{\mathrm{m}}+b_{3}\left(S_{22}^{\mathrm{m}}-S_{44}^{\mathrm{m}}+S_{33}^{\mathrm{m}}\right)\right]$.
These variables apply for the equations for the calculation of the elasticity and compliance tensor of the polycrystal:

$b_{1}=\frac{3}{4} \phi_{0}+\alpha+\beta$,

$b_{2}=\frac{3}{4} \phi_{0}-\alpha+\beta$,

$b_{3}=\frac{1}{4} \phi_{0}-\beta$,

$\alpha=\frac{1}{2} \sin 2 \phi_{0}$,

$\beta=\frac{1}{16} \sin 4 \phi_{0}$.

\section{Appendix B: Equations for calculation of velocities and reflection coefficients}

\section{B1 Velocities in anisotropic media}

To be able to calculate velocities for partial girdle fabric, the calculation of phase velocity for orthorhombic media derived by Daley and Krebes (2004) is used. They rearrange linearised equations to obtain the velocity from an ellipsoidal part an anellipsoidal correction term:

$$
\begin{aligned}
& v_{\mathrm{p}}(\boldsymbol{n})= \\
& \sqrt{1 / \rho\left(C_{11} n_{1}^{2}+C_{22} n_{2}^{2}+C_{33} n_{3}^{2}+2 B_{12} n_{1}^{2} n_{2}^{2}+2 B_{13} n_{1}^{2} n_{3}^{2}+2 B_{23} n_{2}^{2} n_{3}^{2}\right.}, \\
& v_{\mathrm{sv}}(\boldsymbol{n})= \\
& \sqrt{1 / \rho\left(C_{44} \sin ^{2} \psi+C_{55} \cos ^{2} \psi+2 B_{12} n_{1}^{2} n_{3}^{2} \sin ^{2} \psi-2 B_{13} n_{2}^{2} n_{3}^{2}-2 B_{23} n_{1}^{2} n_{3}^{2}\right)}, \\
& v_{\mathrm{Sh}}(\boldsymbol{n})= \\
& \sqrt{1 / \rho\left(C_{44} n_{3}^{2} \cos ^{2} \psi+C_{55} n_{3}^{2} \sin ^{2} \psi+C_{66} \sin ^{2} \vartheta-2 B_{12} n_{1}^{2} \sin ^{2} \psi\right)},
\end{aligned}
$$

with

$$
\begin{aligned}
& B_{12}=\left(C_{13}+2 C_{66}\right)-\left(C_{11}+C_{22}\right) / 2, \\
& B_{13}=\left(C_{12}+2 C_{55}\right)-\left(C_{11}+C_{33}\right) / 2, \\
& B_{23}=\left(C_{23}+2 C_{44}\right)-\left(C_{22}+C_{33}\right) / 2,
\end{aligned}
$$

and the unit phase normal vector

$\boldsymbol{n}=\left(n_{1}, n_{2}, n_{3}\right)=(\sin \vartheta \cos \psi, \sin \vartheta \sin \psi, \cos \vartheta)$,

with the phase angle $\vartheta$ and the azimuth $\psi$, here the azimuth for the orientation of a girdle fabric (Fig. 2).

The components of the group velocity vector are given by (Tsvankin, 2001)

$$
\begin{aligned}
& v_{\mathrm{g}, x_{1}}=v \sin \vartheta+\left.\frac{\partial v_{\mathrm{ph}}}{\partial \vartheta}\right|_{\psi=\text { const }} \cos \vartheta \\
& v_{\mathrm{g}, x_{2}}=\left.\frac{1}{\sin \vartheta} \frac{\partial v_{\mathrm{ph}}}{\partial \psi}\right|_{\vartheta=\mathrm{const}}, \\
& v_{\mathrm{g}, x_{3}}=v_{\mathrm{ph}} \cos \vartheta+\left.\frac{\partial v_{\mathrm{ph}}}{\partial \vartheta}\right|_{\psi=\text { const }} \sin \vartheta .
\end{aligned}
$$


Within the symmetry planes the group velocity can be calculated using $v_{\mathrm{g}, x_{1}}$ and $v_{\mathrm{g}, x_{2}}$ only (Eq. 14).

Outside the symmetry planes of the HTI media the component $v_{\mathrm{g}, x_{2}}$ cannot be neglected as the derivation $\frac{\partial v}{\partial \psi}$ is no longer 0 . In this case $v_{\mathrm{g}}$ is the norm of the group velocity vector $\boldsymbol{v}_{\mathrm{g}}$ considering all three components: $v_{\mathrm{g}, x_{1}}, v_{\mathrm{g}, x_{2}}$ and $v_{\mathrm{g}, x_{3}}$. Here, a second group angle (next to the one in the plane (Eq. 15)) exists for the direction outside the plane with

$\tan \theta_{\text {out }}=\frac{v_{\mathrm{g}, x_{2}}}{\sqrt{v_{\mathrm{g}, x_{1}}^{2}+v_{\mathrm{g}, x_{3}}^{2}}}$.

\section{B2 Reflection coefficients (Zillmer)}

The reflection coefficients as derived by Zillmer et al. (1997, 1998) are given by

$$
\begin{aligned}
R_{\mathrm{pp}}= & \frac{1}{4}\left(\frac{\Delta C_{33}}{C_{44}^{(0)}+2 C_{12}^{(0)}}+\frac{\Delta \rho}{\rho^{(0)}}\right)-\frac{1}{4} \frac{\Delta \rho}{\rho^{(0)}} \tan ^{2}(\vartheta) \\
& +\frac{1}{4} \frac{2 \Delta C_{13}-C_{33}-4 \Delta C 55}{C_{44}^{(0)}+2 C_{12}^{(0)}} \sin ^{2} \vartheta \\
& +\frac{1}{4} \frac{\Delta C_{11}}{C_{44}^{(0)}+2 C_{12}^{(0)}} \sin ^{2} \vartheta \tan ^{2} \vartheta \\
R_{\mathrm{svsv}}= & -\frac{1}{4}\left(\frac{\Delta C_{55}}{C_{12}^{(0)}}+\frac{\Delta \rho}{\rho^{(0)}}\right)-\frac{1}{4} \frac{\Delta \rho}{\rho^{(0)}} \tan ^{2}(\vartheta) \\
& +\frac{1}{4} \frac{\Delta C_{11}-2 \Delta C_{13}+C_{33}-3 \Delta C 55}{C_{12}^{(0)}} \sin ^{2} \vartheta \\
& -\frac{1}{4} \frac{\Delta C_{55}}{C_{12}^{(0)}} \sin ^{2} \vartheta \tan ^{2} \vartheta, \\
R_{\text {shsh }}= & -\frac{1}{4}\left(\frac{\Delta C_{44}}{C_{12}^{(0)}}+\frac{\Delta \rho}{\rho^{(0)}}\right)+\frac{1}{4}\left(\frac{\Delta C_{66}}{C_{12}^{(0)}}+\frac{\Delta \rho}{\rho^{(0)}}\right) \tan ^{2} \vartheta,
\end{aligned}
$$

where $\Delta$ denotes the difference between the upper layer 1 and the lower layer 2, for example $\Delta C_{33}=C_{33}^{(2)}-C_{33}^{(1)}$. The superscript $(0)$ gives the isotropic reference values. When reflection coefficients are calculated for different anisotropic ice fabrics, the density is constant; i.e. the $\Delta \rho$ terms can be neglected $\left(\rho^{(2)}-\rho^{(1)}=0\right)$. 
Acknowledgements. We thank T. Bohlen and his group of the Geophysical Institute, Karlsruhe Institute of Technology, for their support and numerous hints during this study. Financial support for this study was provided to O. Eisen by the Deutsche Forschungsgemeinschaft (DFG) "Emmy Noether" programme grant EI 672/5-1. We thank A. Brisbourne and the anonymous reviewer for their comments which greatly helped to improve the manuscript and H. Gudmundsson for editing of this manuscript.

Edited by: H. Gudmundsson

\section{References}

Aki, K. and Richards, P. G.: Quantitative Seismology, University Science Books Sausalito, California, 2002.

Anandakrishnan, S.: Dilatant till layer near the onset of streaming flow of Ice Stream C, West Antarctica, determined by AVO (amplitude vs offset) analysis, Ann. Glaciol., 36, 283-286, 2003.

Ashby, M. F. and Duval, P.: The creep of polycrystalline ice, Cold Reg. Sci. Technol., 11, 285-300, 1985.

Bass, R., Rossberg, D., and Ziegler, G.: Die elastischen konstanten des Eises, Zeitschr. f. Physik, 149, 199-203, 1957.

Bell, R. E., Ferraccioli, F., Creyts, T. T., Braaten, D., Corr, H., Das, I., Damaske, D., Frearson, N., Jordan, T., Rose, K., Studinger, M., and Wolovick, M.: Widespread Persistent Thickening of the East Antarctic Ice Sheet by Freezing from the Base, Science, 331, 1592-1595, doi:10.1126/science.1200109, 2011.

Bennett, H. F.: An investigation into velocity anisotropy through measurements of ultrasonic wave velocities in snow and ice cores from Greenland and Antarctica, $\mathrm{PhD}$ thesis, University of Wisconsin-Madison, 1968.

Bentley, C. R.: Seismic wave velocities in anisotropic ice: A comparison of measured and calculated values in and around the deep drill hole at Byrd Station, Antarctica, J. Geophys. Res., 77, 44064420, 1972.

Blankenship, D. D. and Bentley, C. R.: The crystalline fabric of polar ice sheets inferred from seismic anisotropy, The Physical Basis of Ice Sheet Modelling (Proceedings of the Vancouver Symposium) IAHS Publ., 54-57, 1987.

Bower, A. F.: Applied mechanics of solids, CRC Press, Boca Raton, Fla., 2010.

Brockamp, B. and Querfurth, H.: Untersuchungen über die Elastizitätskonstanten von See- und Kunsteis, Polarfoschung, 34, $253-$ 262, 1964.

Cuffey, K. M. and Paterson, W. S. B.: The Physics of Glaciers, Elsevier, Butterworth-Heinema, Amsterdam, 4th Edn., 2010.

Daley, P. and Heron, F.: Reflection and transmission coefficients for transversely isotropic media, B. Seismol. Soc. Am., 67, 661-675, 1977.

Daley, P. F. and Krebes, E. S.: Alternative linearized expressions for $\mathrm{qP}, \mathrm{qS} 1$ and $\mathrm{qS} 2$ phase velocities in a weakly anisotropic orthorhombic medium, CREWES Research Report, 16, 1-19, 2004.

Dantl, G.: Die elastischen Moduln von Eis-Einkristallen, Phys. Kondens. Materie, 7, 390-397, 1968.

Diez, A.: Effects of cold glacier ice crystal anisotropy on seismic data, PhD thesis, Karlsruhe Institute of Technology, Germany, 2013.
Diez, A., Eisen, O., Hofstede, C., Bohleber, P., and Polom, U.: Joint interpretation of explosive and vibroseismic surveys on cold firn for the investigation of ice properties, Ann. Glaciol., 54, 201210, 2013.

Diez, A., Eisen, O., Hofstede, C., Lambrecht, A., Mayer, C., Miller, H., Steinahge, D., Binder, T., and Weikusat, I.: Seismic wave propagation in anisotropic ice - Part 2: Effects of crystal anisotropy in geophysical data, The Cryosphere, 9, 385-398, doi:10.5194/tc-9-385-2015, 2015.

Drews, R., Eisen, O., Weikusat, I., Kipfstuhl, S., Lambrecht, A., Steinhage, D., Wilhelms, F., and Miller, H.: Layer disturbances and the radio-echo free zone in ice sheets, The Cryosphere, 3, 195-203, doi:10.5194/tc-3-195-2009, 2009.

Drews, R., Martin, C., Steinhage, D., and Eisen, O.: Characterizing the glaciological conditions at Halvfarryggen ice dome, Dronning Maud Land, Antarctica, J. Glaciol., 59, 9-20, 2013.

Eisen, O., Hamann, I., Kipfstuhl, S., Steinhage, D., and Wilhelms, F.: Direct evidence for continuous radar reflector originating from changes in crystal-orientation fabric, The Cryosphere, 1, 1-10, doi:10.5194/tc-1-1-2007, 2007.

Elvin, A. A.: Number of grains required to homogenize elastic properties of polycrystalline ice, Mechan. Material., 22, 51-64, 1996.

Faria, S. H., Weikusat, I., and Azuma, N.: The microstructure of polar ice. Part II: State of the art, J. Struct. Geol., 61, 21-49, doi:10.1016/j.jsg.2013.11.003, 2014a.

Faria, S. H., Weikusat, I., and Azuma, N.: The microstructure of polar ice. Part I: Highlights from ice core research, J. Struct. Geol., 61, 2-20, doi:10.1016/j.jsg.2013.09.010, 2014b.

Fischer, H., Severinghaus, J., Brook, E., Wolff, E., Albert, M., Alemany, O., Arthern, R., Bentley, C., Blankenship, D., Chappellaz, J., Creyts, T., Dahl-Jensen, D., Dinn, M., Frezzotti, M., Fujita, S., Gallee, H., Hindmarsh, R., Hudspeth, D., Jugie, G., Kawamura, K., Lipenkov, V., Miller, H., Mulvaney, R., Parrenin, F., Pattyn, F., Ritz, C., Schwander, J., Steinhage, D., van Ommen, T., and Wilhelms, F.: Where to find 1.5 million yr old ice for the IPICS "Oldest-Ice" ice core, Clim. Past, 9, 2489-2505, doi:10.5194/cp9-2489-2013, 2013.

Fujita, S., Maeno, H., and Matsuoka, K.: Radio-wave depolarization and scattering within ice sheets: a matrix-based model to link radar and ice-core measurements and its application, J. Glaciol., 52, 407-424, 2006.

Gammon, P. H., Kiefte, H., Clouter, M. J., and Denner, W. W.: Elastic constant of artificial and natural ice samples by brillouin spectroscopy, J. Glaciol., 29, 433-460, 1983.

Graebner, M.: Plane-wave reflection and transmission coefficients for a transversely isotropic solid, Geophysics, 57, 1512-1519, doi:10.1190/1.1443219, 1992.

Green, R. E. and Mackinnen, L.: Determination of the elastic constants of ice single crystals by ultrasonic pulse method, J. Acoust. Soc. Am., 28, 1292, 1956.

Gusmeroli, A., Pettit, E. C., Kennedy, J. H., and Ritz, C.: The crystal fabric of ice from full-waveform borehole sonic logging, J. Geophys. Res., 117, F03021, doi:10.1029/2012JF002343, 2012.

Hill, R.: The elastic behaviour of a crystalline aggregate, Proc. Phys. Soc. A, 65, 349-354, 1952.

Hofstede, C., Eisen, O., Diez, A., Jansen, D., Kristoffersen, Y., Lambrecht, A., and Mayer, C.: Investigating englacial reflections with vibro- and explosive-seismic surveys at Halvfarryggen ice dome, Antarctica, Ann. Glaciol., 54, 189-200, 2013. 
Horgan, H. J., Anandakrishnan, S., Alley, R. B., Peters, L. E., Tsoflias, G. P., Voigt, D. E., and Winberry, J. P.: Complex fabric development revealed by englacial seismic reflectivity: Jakobshavn Isbræ, Greenland, Geophys. Res. Letters, 35, L10501, doi:10.1029/2008GL033712, 2008.

Horgan, H. J., Anandakrishnan, S., Alley, R. B., Burkett, P. G., and Peters, L. E.: Englacial seismic reflectivity: imaging crystalorientation fabric in West Antarctica, J. Glaciol., 57, 639-650, 2011.

Jona, F. and Scherrer, P.: Die elastischen Konstanten von EisEinkristallen, Helvetica Phys. Acta, 25, 35-54, 1952.

Keith, C. M. and Crampin, S.: Seismic body waves in anisotropic media: reflection and refraction at a plane interface, Geophys. J. R. Astr. Soc., 49, 181-208, 1977.

Kohnen, H.: The temperature dependence of seismic waves in ice, J. Glaciol., 13, 144-147, 1974.

Mainprice, D., Hielscher, R., and Schaeben, H.: Calculating anisotropic physical properties from texture data using the MTEX open-source package, Geological Society, London, Special Publications, 360, 175-192, doi:10.1144/SP360.10, 2011.

Marín, C., Gudmundsson, G. H., Pritchard, H. D., and Gagliardini, O.: On the effects of anisotropic rheology on ice flow, internal structure, and the age-depth relationship at ice divides, J. Geophys. Res., 114, 1-18, 2009a.

Martín, C., Hindmarsh, R. C. A., and Navarro, F. J.: On the effects of divide migration, along-ridge flow, and basal sliding on isochrones near an ice divide, J. Geophys. Res.-Earth Surf., 114, F02006, doi:10.1029/2008JF001025, 2009b.

Matsuoka, K., Furukawa, T., Fujita, S., Maeno, H., Urantsuka, S., Naruse, R., and Watanabe, O.: Crystal orientation fabrics within the Antarcitc ice sheet revealed by a mutlipolarization plane and dual-frequency radar survey, J. Geophys. Res., 108, 2499, doi:10.1029/2003JB002425, 2003.

Matsuoka, K., Wilen, L., Huerly, S. P., and Raymond, C. F.: Effects of birefringence within ice sheets on obliquely propagating radio waves, IEEE Trans. Geosci. Remote Sens., 47, 1429-1443, 2009.

Nanthikesan, S. and Sunder, S. S.: Anisotropic elasticity of polycrystalline ice Ih, Cold Reg. Sci. Technol., 22, 149-169, 1994.

NEEM community members: Eemian interglacial reconstructed from a Greenland folded ice core, Nature, 493, 489-494, doi:10.1038/nature11789, 2013.

Penny, A. H. A.: A theoretical determination of the elastic constants of ice, Mathematical Proceedings of the Cambridge Philosophical Society, 44, 423-439, 1948.

Peternell, M., Hasalova, P., Wilson, C. J. L., Piazolo, S., and Schulmann, K.: Evaluating quartz crystallographic preferred orientations and the role of deformation partitioning using EBSD and fabric analyser techniques, J. Struct. Geol., 32, 803-817, 2010.

Peters, L. E., Anandakrishnan, S., Holland, C. W., Horgan, H. J., Blankenship, D. D., and Voigt, D. E.: Seismic detection of a subglacial lake near the South Pole, Antarctica, Geophys. Res. Letters, 35, L23501, doi:10.1029/2008GL035704, 2008.

Pettit, E. C., Thorsteinsson, T., Jacobson, H. P., and Waddington, E. D.: The role of crystal fabric in flow near an ice divide, J. Glaciol., 53, 277-288, doi:10.3189/172756507782202766, 2007.
Polom, U., Hofstede, C., Diez, A., and Eisen, O.: First glaciervibroseismic experiment-results from the cold firn of Colle Gnieftti, Near Surf. Geophys., 12, 493-504, doi:10.3997/18730604.2013059, 2014.

Raymond, C. F.: Deformation in the vicinity of ice divides, J. Glaciol., 29, 357-373, 1983.

Reuss, A.: Berechnung der Fliessgrenzen von Mischkristallen auf Grund der Platizitätsbedingung für Einkristalle, Zeitschr. Angew. Math. Mech., 9, 49-58, 1929.

Rommel, B. E. and Tsvankin, I.: Analytic description of P-wave ray direction and polarization in orthorhombic media, in: Anisotropy 2000: fractures, converted waves, and case studies, edited by: Ilkelle, L. T., 1-19, Society of Exploration Geophysicists, 2000.

Rüger, A.: P-wave reflection coefficents for transversely isotropic models with vertical and horizontal axis of symmetry, Geophysics, 62, 713-722, 1997.

Rüger, A.: Reflection Coefficients and Azimuthal Avo Analysis in Anisotropic Media, Geophysical Monograph Series, No. 10, Society of Exploration Geohysicists, 2002.

Smith, A. M.: Subglacial bed properties from normal-incidence seismic reflection data, J. Environ. Eng. Geophys., 12, 3-13, 2007.

Sunder, S. S. and Wu, M. S.: Crack nucleation due to elastic anisotropy in polycrystalline ice, Cold Reg. Sci. Technol., 18, 29-47, 1994.

Thomsen, L.: Weak anisotropic reflections, Vol. 8 of Offsetdependent reflectivity - Theory and Practice of AVO Analyses, Investigations in Geophysics Series, Society of Exploration Geophysicists, 1993.

Tsvankin, I.: Seismic signatures and analysis of reflection data in anisotropic media, Vol. 29 of Handbook of geophysical exploration, seismic expolration, Pergamon, 2001.

Voigt, W.: Lehrbuch der Kristallphysik: (mit Ausschluss der Kristalloptik), Bibliotheca mathematica Teubneriana; 12, Johnson, New York, 1910.

Wallbrecher, E.: Tektonische und Gefügeanalytische Arbeitsweisen, Ferdinand Enke Verlag, Stuttgart, 1986.

Wilson, C. J. L., Russell-Head, D. S., and Sim, H. M.: The application of an automated fabric analyzer system to the textural evolution of folded ice layers in shear zones, Ann. Glaciol., 37, 7-17, 2003.

Woodcock, N. H.: Specification of fabric shapes using an eigenvalue method, Geol. Soc. Am. Bull., 88, 1231-1236, 1977.

Zillmer, M., Gajewski, D., and Kashtan, B. M.: Reflection coefficients for weak anisotropic media, Geophys. J. Int., 129, 389398, 1997.

Zillmer, M., Gajewski, D., and Kashtan, B. M.: Anisotropic reflection coefficients for a weak-contrast interface, Geophys. J. Int., 132, 159-166, 1998. 